\title{
Using semi-open questions to integrate perceptions in choice models
}

\author{
Aurélie Glerum * \\ Bilge Atasoy* \\ 13th December 2013 \\ Report TRANSP-OR 120325 \\ Transport and Mobility Laboratory \\ Ecole Polytechnique Fédérale de Lausanne \\ transp-or.epfl.ch
}

Michel Bierlaire *

\begin{abstract}
This research investigates the measurement of perceptions by means of adjectives freely reported by respondents in semi-open questions. It involved the use of semi-open responses of 1'763 Swiss individuals to develop indicators for a latent variable representing the perception of comfort of public transportation. The indicators are then incorporated into a discrete choice model of revealed mode choices.

Perceptions are assumed to impact choice significantly and this research aims at capturing their complexity using adjectives and integrating them into the hybrid choice modeling framework.

We exploit a quantification of the adjectives performed by external evaluators. Given the subjectivity that is involved, we analyze the sensitivity of the results across evaluators who rated the adjectives. We observe that the aggregate indicators of demand, such as market shares, elasticities and values of time, are rather robust across evaluators. This is not the case for the disaggregate indicators that may vary substantially across evaluators.
\end{abstract}

\section{Key words}

Mode choice models, latent variables, perceptions, qualitative data, hybrid choice models.

*ÉCOLE POLYTECHNIQUE FÉDÉRALE DE LAUSANNE (EPFL), School of Architecture, Civil and Environmental Engineering (ENAC), Transport and Mobility Laboratory (TRANSP-OR), \{aurelie.glerum, bilge.kucuk, michel.bierlaire\}@epfl.ch 


\section{Introduction}

Recently the discrete choice modeling (DCM) literature has been influenced by the development of new models, namely the hybrid choice models (HCM). Among other properties, such models allow for the integration of psychological constructs such as perceptions as explanatory variables of the choice. The recent motivation for the integration of perceptional aspects into discrete choice models has raised a few issues regarding their measurement. First, it is essential to develop an adequate way to quantify measures of the perceptions and integrate them into a discrete choice model. Second, the subjectivity inherent to the quantification procedure must be assessed. The present research aims at addressing these issues.

To evaluate psychological constructs such as perceptions, attitudes or lifestyles, survey techniques have been developed by social scientists and involve the collection of psychometrics (Thorndike, 1920; Likert, 1932; Bearden and Netemeyer, 1999). They usually consist of proposing statements where respondents have to indicate a rating on a five-point scale. For example, Vredin Johansson et al. (2006) collect ratings of the importance of some perceptional questions related to comfort, convenience and flexibility of transport modes. Abou-Zeid et al. (2012) ask respondents to indicate their satisfaction when they commute either by public transportation or by car. Atasoy et al. (2013) request individuals to rate their agreement on a list of statements related to environmental concern, mobility, residential choice or lifestyle. The survey methods used for these studies mostly rely on closed questions.

The main advantage of such questions is that they are easy to collect, code and integrate into existing model frameworks. However, social scientists emphasize on the importance of exploiting the information contained in responses to open questions. Such questions are often underexploited or only used as a complement to closed questions (Looker et al., 1989). Mossholder et al. (1995) point out the importance of such questions to retrieve constructs that can be recognized in the semantic content of language, such as affection or emotions. In particular, Beirao and Cabral (2007) use open questions to measure attitudes towards public transportation and car. More importantly, it is recognized that such questions reflect better respondents' conception of a construct (Potkay and Allen, 1973; Mossholder et al., 1995; Kaufmann et al., 2001), while closed questions are the result of the survey designer's representation of it. The diversity of answers in open questions is also wider than in closed questions (Schuman and Presser, 1996).

Open questions can be structured in various ways. In this research we focus on a subset of them, which we denote by semi-open questions, and where we ask the respondents to report several adjectives describing best a variable of interest (e.g. a transportation mode). This data 
collection technique has been used in psychology to obtain a representation of a person (Potkay and Allen, 1973) and more recently in social sciences to obtain representations of transportation modes (Kaufmann et al., 2001, 2010). Though this data collection method and the well-known word association technique may show some common features, we distinguish them. In word association surveys, individuals are required to report the 'first single word which comes to mind' (Cramer, 1968) when they are exposed to a stimulus word. In semi-open questions, respondents are required to reply to a more precise question and give a more structured answer.

The use of semi-open questions to measure psychological constructs such as perceptions requires the development of an appropriate modeling framework. For behavioral researchers, the heterogeneity in individuals' behavior has always been an interesting field and several methodologies are developed in order to account for this heterogeneity. In transportation research, mixed logit models are used to capture the random taste heterogeneity across consumers (McFadden and Train, 2000; Hess and Train, 2011). Mixed logit models are flexible in terms of the underlying distribution assumptions for the unobserved heterogeneity (Greene and Hensher, 2003). On the other hand, many researchers work with latent variables (LV) / latent classes (LC) in order to explain the heterogeneity with structural equation models. These structural equation models are built with the characteristics of individuals in order to obtain a systematic representation of the heterogeneity. The latent constructs are integrated into choice models using the HCM framework (Walker, 2001; Walker and Ben-Akiva, 2002; Ben-Akiva et al., 2002). In the literature, the effect on choice of LVs measured by closed questions has been evaluated for various applications. In transportation mode choice contexts, Espino et al. (2006) assess the effect of the LV 'comfort' on the choice between bus and car. Vredin Johansson et al. (2006) also analyze the impact of comfort on mode preferences as well as the one of flexibility and care for the environment. Daly et al. (2012) model the effect of security on rail travel behavior.

In this paper, we consider the HCM framework and illustrate our methodology by modeling the impact of the LV 'perception of comfort of public transportation' on choice. Since we are using responses to semi-open questions as measurements of the $\mathrm{LV}$, a method to quantify the responses to the semi-open questions is required. We propose a method which consists of asking a set of individuals (the 'evaluators') to rate the adjectives on a scale relative to the LV, where a positive number indicates a positive representation of the LV and a negative number implies a negative representation of it. The use of individuals to evaluate qualitative concepts is common practice in computer science studies (Amazon's Mechanical Turk, 2005-2012; Franklin et al., 2011) and research on natural language processing (Snow et al., 2008). Humans happen to be more effective than computers for some specific tasks, such as evaluating a language (Snow et al., 2008; Sorci 
et al., 2010; Robin et al., 2011; Venetis et al., 2012). Moreover, in medical and social sciences scores from a number of raters are used for decision-making. In such domains it is critical to have an agreement between the raters in order to be fair to the subjects. Therefore there is a well-studied literature on how to minimize the impact of the rater factor on the data quality. We refer to the review by Banerjee et al. (1999) for the analysis of agreement between raters which is called as inter-rater reliability. However in our case, we are interested in the heterogeneity of raters and we analyze the impact of different ratings on the results. Therefore we do not seek for inter-rater reliability but rather analyze the differences between ratings.

To assess the sensitivity of the market shares of the different transportation modes to variations of exogenous factors, different demand indicators need to be derived. In a transportation mode choice context, important demand indicators include willingness-to-pay (WTP) indicators, such as the value of time (VOT), and elasticities. In the literature, it has been shown that HCMs allow for a more complex representation of the demand. For example, Abou-Zeid et al. (2010) capture the heterogeneity in the VOT within the population by considering an interaction term between travel time and the LV. Bolduc and Daziano (2011) show that several other WTP indicators can be derived to valuate the demand in a vehicle choice context. Yáñez et al. (2010) analyze the effect of changes in the LV on individuals' choices. The variations are indeed triggered by changes in an explanatory variable of the structural model of the LV. Building upon the ideas of Abou-Zeid et al. (2010) and Yáñez et al. (2010), we respectively derive a series of demand indicators and analyze the effect of a change in an explanatory variable of comfort of public transportation on the choice. The novelty of our approach consists of considering the distribution of the indicators across evaluators and providing statistics on it. We hence aim at demonstrating the robustness of the method in terms of model application, when the ratings of different evaluators are considered.

In this paper, we achieve a quantification of data from adjectives and introduce this information as measurement of an LV in an HCM. We moreover propose an analysis of the sensitivity of the demand indicators to the evaluators' ratings, which leads to the empirical evidence that disaggregate indicators of demand vary more importantly across evaluators than aggregate indicators.

This research is illustrated by a case study which aims at analyzing individuals' transportation mode choice in low-density areas of Switzerland. A revealed preferences (RP) survey was conducted in the context of a joint project between PostBus, one of the major companies operating in such regions, and EPFL's Transportation Center (TraCe). In this survey, inhabitants were asked to describe all trips performed on a particular day as well as a the chosen transport modes. Moreover, they were asked to report adjectives which describe best a list of transportation modes. The field of possible answers for such questions is wide and therefore allows for the analysis of a variety 
of themes related to perception. For instance, one could model perception of flexibility, environmental impact or reliability of a particular transport mode. In this paper the analysis of the impact on choice of individuals' perception of comfort of public transportation is presented. For previous work on this research, we refer to Glerum et al. (2011).

The paper is structured as follows. Section 2 presents the data collection phase. Section 3 describes the specification of the HCM and explains how qualitative adjective data are integrated into the framework. Section 4 presents the estimation results. Section 5 presents a validation of the HCM. Section 6 presents a forecasting analysis by means of demand indicators derived for all evaluators. Section 7 discusses the implications of the present data collection and modeling approach on future questionnaire designs. Section 8 concludes by presenting some possible further developments.

\section{Data collection}

In order to analyze the transportation mode choices of inhabitants of regions which are loosely connected, an RP survey was conducted between 2009 and 2010. Two copies of a questionnaire were sent to each household of 57 towns or villages connected by post busses. The towns and villages were selected in order to be representative of the whole network of PostBus and respondents of 16 years and over were asked to answer the questionnaire. Individuals had to answer the survey in German or French, depending on the language region they were living in. In total, 20'138 households were surveyed and 1'763 valid questionnaires from 1'326 different households were eventually collected. Further details about the data collection procedure can be found in Bierlaire et al. (2011).

The following sections present the structure of the survey and the quantification process of the indicators of perception.

\subsection{Revealed preferences survey}

In the RP survey, information was collected on all the trips respondents performed in one day, including the mode(s) they used, the trip duration and the cost of fuel or public transport ticket, on their opinions on a list of statements, on their mobility habits, household structure and socioeconomic information. In total, $2^{\prime} 265$ trips were identified.

In addition, respondents were questioned about their perceptions of transport modes. Precisely, they were asked to report three adjectives describing best each transport mode of a given list. The answers to these questions provide spontaneous indicators of individuals' perceptions, since they 
consist of words which they freely reported. We refer to these type of questions as semi-open questions.

Let us note that due to the inaccuracy of the durations and costs reported by the respondents for each of their trips, we imputed times and costs based on the websites of the Swiss railways (SBB) http://www.cff.ch and of ViaMichelin http://fr.viamichelin.ch. The same websites were used to impute the times and costs for the non-chosen alternatives. For the travel cost, the marginal cost of the trip is considered. More precisely, for car/motorbike trips, the only considered costs are the fuel costs for the associated trip. Similarly for public transport alternatives, the cost of the trip is calculated by accounting for the possible ownership of passes, i.e. for individuals that do not own any pass, this cost is set to the standard trip fare indicated by the SBB website, while for individuals who do own one, it is set to 0 . However costs of subscriptions to the PT system (i.e. yearly passes, monthly passes, etc.) are ignored.

In addition to the imputation of the travel time and costs, we aggregated the modes reported by the individuals in three groups: public transportation modes, private motorized modes and soft modes (see Section 3 for the description). This aggregation was done since the interest of the paper is to take into account the perceptions of individuals in order to better understand their preferences towards public, private and soft modes, rather than the specific choices of train, bus, motorbike, etc.

Some bias occurred in the responses to the survey. In particular, individuals with a high education level, with ages between 40 and 79 years, or men had a higher response rate than other categories. To evaluate the demand for the different transportation modes, we correct the response bias by introducing a sample weight $w_{n}$ for each individual $n$ in the computation of the indicators. These weights are computed using the iterative proportional fitting (IPF) algorithm. This procedure enables us to make our sample representative of the population with respect to its marginal distributions of education, age and gender. We have made the assumption that self-selection only occurs according to exogenous socio-economic characteristics. However it could also occur in the response behavior, implying that individuals using public transportation may show a higher response rate than car users (Brownstone, 1998). For that purpose, we would also need to weight the sample according to the transport mode shares in the Swiss population. We leave this for future research.

More detailed information on the survey can be found in Atasoy et al. (2013). 


\subsection{Semi-open questions}

In the section of the questionnaire on the perception of the transport modes, respondents had to report three adjectives describing best the following set of transport modes: car, train, bus/metro/tram, post bus, bike or walk. As an illustration, Table 1 presents the semi-open questions as they appeared in the questionnaire.

For each of the following transport modes, give three adjectives that describe them best according to you.

\begin{tabular}{|l|l|l|l|l|}
\cline { 3 - 5 } \multicolumn{2}{l|}{} & Adjective 1 & Adjective 2 & Adjective 3 \\
\hline 2 & The car is: & & & \\
\hline 3 & The bus, the metro and the tram are: & & & \\
\hline 4 & The post bus is: & & & \\
\hline 5 & The bicycle is: & & & \\
\hline 6 & The walk is: & & & \\
\hline
\end{tabular}

Table 1: Semi-open questions on the perception of several transport modes.

With the help of social scientists, we grouped these adjectives into several themes including comfort, perception of time, perception of cost, difficulty of access, flexibility, efficiency, reliability, environmental impact, appreciation, feeling or look. The adjectives classified within each theme provide information which is assumed to reflect closely each respondent's perception of each topic, as they are freely reported.

In the model presented in this paper (see Section 3 for the specification), we are interested in evaluating the effect of the perception of one of the characteristics of transport modes listed above, that is comfort of public transportation, on the mode choice. Hence, we use the adjective data classified in the theme of comfort and relative to modes train, bus/metro/tram and post bus as indicators of this particular attribute. As respondents reported three adjectives for each of the three public transportation alternatives we have at most nine indicators of the perception of comfort of public transportation per individual. The variable of comfort of public transportation was selected due to the fact that a large number of related adjectives were identified in the procedure of classification into themes.

About $16 \%$ of the answers to the nine indicators were classified as related to comfort (see Table 2). Despite this fairly high proportion of relevant adjectives, about $10 \%$ of the respondents 
who provided answers to the semi-open questions did not provide any adjective related to comfort (see Table 3). These 10\% cases are hence lost and will not provide information to the model.

\begin{tabular}{r|r} 
& Percentage \\
\hline Related to comfort & 15.58 \\
Unrelated to comfort & 53.80 \\
Missing & 30.62 \\
\hline
\end{tabular}

Table 2: Percentages of adjectives related to comfort, unrelated to comfort and missing, among the responses to the 9 indicators related to public transportation (

\begin{tabular}{l|rrrrrrrrrrr}
$\begin{array}{l}\text { Number of adjectives } \\
\text { unrelated to comfort }\end{array}$ & 0 & 1 & 2 & 3 & 4 & 5 & 6 & 7 & 8 & 9 \\
\hline Percentage & 0.05 & 2.80 & 4.90 & 7.75 & 8.18 & 12.21 & 22.05 & 18.45 & 13.61 & 10.01 \\
\hline
\end{tabular}

Table 3: Percentages of $0, \ldots, 9$ adjectives unrelated to comfort, among the responses to the 9 indicators related to public transportation (sample size $=2^{\prime} 265$ ). Missing values are not considered in the computation of the percentages.

In order to use the nine indicators as a measure of the perception of comfort, they need to be quantified, i.e. we need to find a scale of 'comfort'. Therefore we designed another survey dedicated to the quantification of the adjectives.

\subsection{Ratings of 'evaluators'}

We conducted an online survey for the quantification of all adjectives which were identified to be related to comfort. We asked 25 students and employees of EPFL to fill it in. Since an important proportion of these individuals were neither speaking German nor French, the adjectives were translated into English. We are aware that the translation of the adjectives might generate a bias and leave this problem for future research. The exhaustive list of adjectives related to comfort is presented in Table $4^{1}$. The students and employees of EPFL were requested to rate how strongly each adjective characterizes the concept of comfort on a five-point scale from -2 to 2 . A positive rating means that the evaluator associates the adjective with being comfortable and a negative rating corresponds to discomfort. The evaluators were asked to report 0 if the adjective is not related to the concept of comfort in transport modes.

In Table 4 we report basic statistics on the reported ratings by the 25 evaluators. On the one hand, some of the adjectives are perceived in a similar way by the evaluators such as comfortable

\footnotetext{
${ }^{1}$ Some of the words reported by respondents are actually not adjectives. They were nevertheless included in the analysis.
} 
having a lower standard deviation compared to others. On the other hand, there are some adjectives with a standard deviation higher than 1 such as hardly full and packed. Such ambiguities are the main driving force of this study for the quantification of indicators of perceptions.

For illustration, we selected two evaluators among the 25 , using the following procedure based on the Euclidean distances between the ratings of the evaluators. The first one, called the central evaluator, is selected such that the maximum distance from all other evaluators is the smallest. The other evaluator (outlying evaluator) is the one that is the furthest from the central evaluator. We report the ratings of these two evaluators in Table 4. It is observed that the central evaluator is closer to the mean value compared to the outlying evaluator as expected. The outlying evaluator has a different perception of the adjectives in terms of their positive/negative characterization of comfort. In Section 4 we present the estimation results for these two evaluators to see the effect of different ratings on the estimated parameters. We also include the estimation results of a model using the median ratings of the adjectives. These median ratings are provided in the third column of Table 4. Furthermore, in Section 6 we provide a sensitivity analysis of demand indicators including market shares, elasticities and VOTs, across all 25 evaluators. 


\begin{tabular}{l|ccl|cc} 
Adjectives & Mean & Median & $\begin{array}{l}\text { Standard } \\
\text { deviation }\end{array}$ & $\begin{array}{c}\text { Central } \\
\text { evaluator }\end{array}$ & $\begin{array}{c}\text { Outlying } \\
\text { evaluator }\end{array}$ \\
\hline bad air & -1.52 & -2 & 0.714 & -1 & 1 \\
bumpy & -1.08 & -1 & 0.862 & -1 & 1 \\
comfortable & 1.72 & 2 & 0.542 & 1 & 2 \\
difficult & -1.00 & -1 & 0.707 & -1 & -2 \\
empty & 0.880 & 1 & 0.726 & 1 & 1 \\
expensive & -0.680 & 0 & 0.988 & -2 & -1 \\
fast & 1.04 & 1 & 0.735 & 2 & 1 \\
full & -1.00 & -1 & 1.00 & -2 & 2 \\
hard & -0.920 & -1 & 0.640 & -1 & -1 \\
hardly full & -0.280 & 0 & 1.28 & 1 & 2 \\
irritating & -1.44 & -2 & 0.870 & -1 & 1 \\
packed & -0.880 & -1 & 1.20 & -2 & 1 \\
relaxing & 1.72 & 2 & 0.737 & 1 & 1 \\
restful & 1.44 & 2 & 0.821 & 1 & 2 \\
shaking & -1.08 & -1 & 0.997 & -1 & 1 \\
stressful & -1.44 & -2 & 0.917 & -1 & 1 \\
suffocating & -1.60 & -2 & 0.817 & -1 & 1 \\
tiring & -1.16 & -1 & 0.800 & -1 & 2 \\
uncomfortable & -1.56 & -2 & 0.870 & -1 & -1 \\
unsuitable with bags & -0.920 & -1 & 0.812 & -1 & 2 \\
unsuitable with strollers & -0.720 & -1 & 0.891 & 0.866 & -1 \\
without stress & 1.40 & 2 & & -1 \\
\hline
\end{tabular}

Table 4: Basic statistics on the ratings of evaluators ( sample size $=25$ ).

\section{Model specification}

The choice is defined as the transport mode used by the respondents. It is one of the three following categories:

- Public transportation (PT) modes, such as bus, train, etc. 
- Private motorized modes (PMM), such as car, motorbike, etc.

- Soft modes (SM), such as walk or bike.

The respondents' mode choices are analyzed on tours and not on single trips, that is, one observation corresponds to one sequence of trips starting from each respondent's home and ending at the same place. For example, one simple tour can consist of a sequence of starting points and destinations home-work-home. A longer tour such as home-work-shopping-home can include an additional trip for shopping.

Hence, the choice variable is defined as the set of modes, i.e. PT, PMM or SM, used on each tour.

In the DCM, the deterministic parts of the utility functions are given as follows:

$$
\begin{aligned}
& V_{\mathrm{PMM}}=\mathrm{ASC}_{\mathrm{PMM}}+\beta_{\mathrm{cost}} \cdot \operatorname{cost}_{\mathrm{PMM}}+\beta_{\mathrm{time} \mathrm{PMM}_{\mathrm{PM}}} \cdot \operatorname{time}_{\mathrm{PMM}}+\beta \text { work }_{\mathrm{PMM}} \cdot \text { work } \\
& +\beta_{\text {French }_{\text {PMM }}} \cdot \text { French } \\
& V_{\mathrm{PT}}=\mathrm{ASC}_{\mathrm{PT}}+\beta_{\text {cost }} \cdot \operatorname{cost}_{\mathrm{PT}}+\beta_{\text {time }_{\mathrm{PT}}} \cdot \text { time }_{\mathrm{PT}}+\beta \text { work }_{\mathrm{PT}} \cdot \text { work } \\
& +\beta_{\text {French }_{\mathrm{PT}}} \cdot \text { French }+\beta_{\text {comfort }} \cdot \mathrm{PCPT} \cdot \text { time }_{\mathrm{PT}} \cdot \frac{1}{1000} \\
& V_{\mathrm{SM}}=\beta_{\text {distance }} \cdot \text { distance }
\end{aligned}
$$

- We assume that the deterministic utilities $V_{\mathrm{PMM}}$ and $V_{\mathrm{PT}}$ are influenced by the travel times time $_{\mathrm{PMM}}$ and time $_{\mathrm{PT}}$, and travel costs $\operatorname{cost}_{\mathrm{PMM}}$ and $\operatorname{cost}_{\mathrm{PT}}$. For the $\mathrm{SM}$ alternative, a distance term distance is included.

- In addition to the characteristics of the transportation alternatives, some socio-economic variables are assumed to have an impact on the transportation mode choice, that is, variable work which indicates that the respondent performed home-work-home tours and variable French which indicates that the respondent resides in a French-speaking region of Switzerland. The latter is introduced into the model since the public transportation offer is slightly better in German-speaking regions than in the French-speaking ones, hence generating differences in the demand for the three types of modes. For example, the average number of daily return trips of suburban busses is 17 in French-speaking regions versus 29 in Germanspeaking regions (Bierlaire et al., 2011; p. 45).

- In the deterministic utility $V_{\mathrm{PT}}$, a latent explanatory variable $P C P T$ is also included and accounts for the image people have of comfort of PT. It is specified as an interaction with the travel time variable in order to analyze the impact of the LV on sensitivity to travel time. The main effect of LV PCPT is not included in the utility function, since its integration led 
to the non-significance of the interaction term. Though a model including the main effect alone would be the usual selection (due to the parsimony of its specification), the model with the interaction term is adopted for the purposes of illustrating how taste heterogeneity in the sensitivity to travel time can be captured.

To avoid estimation problems that may arise when variables fall into very different ranges, we divide the time by 1000 in the interaction term.

The perception of comfort of PT PCPT is described by the following structural equation:

$$
\begin{aligned}
\text { PCPT } & =\lambda_{\text {mean }}+\lambda_{\text {French }} \cdot \text { French }+\lambda_{\text {age }_{50}} \cdot \text { age }_{50}+\lambda_{\text {active }} \cdot \text { active } \\
& +\lambda_{\text {cars }} \cdot \text { cars }+\omega, \quad \text { with } \omega \sim \mathscr{N}\left(0, \sigma^{2}\right) .
\end{aligned}
$$

In the above equation we specify an intercept $\lambda_{\text {mean }}$ and assume that several socio-economic variables have an effect on an individual's perception of comfort of PT:

- An indicator French of a residence in a French-speaking region;

- A variable $a g e_{50}$ indicating that the respondent is younger than 50 years;

- A variable active equal to 1 if the respondent has a full-time or part-time job, and 0 for any other working status;

- A variable cars indicating that the respondent's household owns at least 2 cars;

A normally distributed random variable $\omega$ with mean 0 and standard deviation $\sigma$ is also added as an error term.

The inclusion of the above variables in the structural equation of the latent variable model (LVM) results from an iterative model building procedure. The presented specification is hence the best which we eventually reached.

As the LV PCPT cannot be directly quantified by a survey question, measurement equations that relate it with indicators are specified. The indicators are the values ranging from -2 to 2 which were assigned to each adjective by the evaluators as explained in Section 2.3. The measurement equation for each of the nine indicators $I_{k}$, with $k=1, \ldots, 9$ is specified as follows:

$$
I_{k}=\alpha_{k}+\gamma_{k} \cdot \mathrm{PCPT}+v_{k}, \quad \text { with } v_{k} \sim \mathscr{N}\left(0, \sigma_{k}^{2}\right) \text {, }
$$

where $\alpha_{k}, \gamma_{k}$ and $\sigma_{k}$ are parameters to be estimated. 


\section{Estimation results}

Parameters $A S C_{h}$, with $h \in\{\mathrm{PMM}, \mathrm{PT}\}, \beta_{i}$, with $i \in\left\{\right.$ cost $_{\text {time }}$ tim , time $_{P T}$, distance, work $_{P M M}$, work $_{P T}$, French $_{P M M}$, French $_{P T}$, PCPT $\}, \lambda_{j}$, with $j \in\{$ mean, French, age 50 , active, cars $\}, \sigma, \alpha_{k}, \gamma_{k}$ and $\sigma_{k}$ with $k=1, \ldots, 9$ are estimated using exogenous sample maximum likelihood (ESML) (Ben-Akiva and Lerman, 1985) using the Python version of the software Biogeme (Bierlaire and Fetiarison, 2009). The following likelihood function is considered:

$$
\mathscr{L}=\prod_{n=1}^{N} \int_{\mathrm{PCPT}} P\left(y_{n} \mid X_{n}, \mathrm{PCPT}_{n} ; \beta\right)\left\{\prod_{k=1}^{9} f\left(I_{k n} \mid \mathrm{PCPT}_{n} ; \alpha_{k}, \gamma_{k}, v_{k}, \sigma_{k}\right)\right\} f\left(\mathrm{PCPT}_{n} ; \lambda, \sigma\right) d \mathrm{PCPT},
$$

where $N$ is the total sample size, $X_{n}$ is a vector of socio-economic attributes of respondent $n, y_{n}$ is a vector of choice indicators for $n$, such that $y_{i n}=1$, if individual $n$ selects alternative $i$, and $y_{i n}=0$ otherwise. In the likelihood formula, $P\left(y_{n} \mid X_{n}, \mathrm{PCPT}_{n} ; \beta\right)$ is the choice probability of $n$, $f\left(I_{k n} \mid \mathrm{PCPT}_{n} ; \alpha_{k}, \gamma_{k}, v_{k}, \sigma_{k}\right)$ is the value of the density function of $I_{k}$ for $n, f\left(\mathrm{PCPT}_{n} ; \lambda, \sigma\right)$ is the value of the density function of $P C P T$ for $n$.

Let us note that for identification purposes, $\alpha_{1}$ is fixed to 0 and the standard deviation $\sigma$ of the LV is normalized to 1 . Moreover, missing values of $I_{k n}$ do not contribute to the likelihood.

The HCMs are estimated separately for all the 25 evaluators (see Tables 10, 11 and 12 in the Appendix for the estimation results of all HCMs). All components of the HCMs are estimated simultaneously. In Table 5 we provide the results for the two evaluators identified in Section 2.3 and the results for the model using the median ratings of the adjectives. These three sets of results were selected for presentation since they give an idea on the impact of the ratings of adjectives on the estimation results taking into account the extreme and median ratings. A first observation is that, although the estimated parameters are close for different evaluators, the results with outlying evaluator differs in a non-negligible way from the other sets of results for some parameters. The parameters that differ the most from the other evaluators are the ones that are closely related to the ratings of adjectives, namely the parameters associated with the explanatory variables of the LV. As explained in Section 2.3 the ratings of the outlying evaluator differ in terms of both the sign and the magnitude from the other evaluators.

In addition to the estimation results of the three selected evaluators, we also display the estimation of a logit model, which is a function of the same variables as the ones contained in the structural equations of the HCMs. More precisely, the utilities of the logit model are the same as in the HCMs, except for the PT alternative, which is expressed as follows: 


$$
\begin{aligned}
& V_{\mathrm{PT}}=\mathrm{ASC}_{\mathrm{PT}}+\beta_{\mathrm{cost}} \cdot \operatorname{cost}_{\mathrm{PT}}+\beta_{\text {time }_{\mathrm{PT}}} \cdot \text { time }_{\mathrm{PT}}+\beta \text { work }_{\mathrm{PT}} \cdot \text { work } \\
& +\beta_{\text {French }_{\mathrm{PT}}} \cdot \text { French }+\lambda_{\text {French }} \cdot \text { French } \cdot \text { time }_{\mathrm{PT}} \cdot \frac{1}{1000}+\lambda_{\mathrm{age}_{50}} \cdot \text { age }_{50} \cdot \text { time }_{\mathrm{PT}} \cdot \frac{1}{1000} \\
& +\lambda_{\text {active }} \cdot \text { active } \cdot \text { time }_{\mathrm{PT}} \cdot \frac{1}{1000}+\lambda_{\text {cars }} \cdot \text { cars } \cdot \text { time }_{\mathrm{PT}} \cdot \frac{1}{1000}
\end{aligned}
$$

The following conclusions can be drawn from the estimates of the choice model component of the HCMs:

- The negative signs of the parameters $\beta_{\text {cost }}, \beta_{\text {distance }}, \beta_{\text {time }}$ PMM and $\beta_{\text {timept }}$ are consistent with expectations. Since the travel time of PT is interacted with the LV, the time coefficient differs from one evaluator to the other. It also differs from the logit model.

- Some socio-economic variables have a significant effect on the choice of transport modes. First, performing tours that only include a trip from home to work and back from work to home decreases the probability of choosing PMM relative to the base alternative of SM.

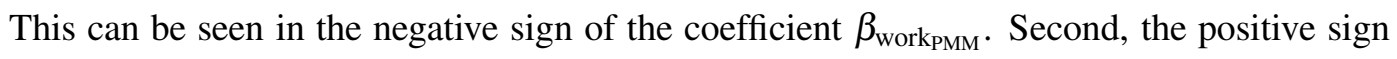
of $\beta_{\text {FrenchPMM }}$ shows that being from a French-speaking region increases the probability of choosing PMM relative to SM. For PT we cannot draw clear conclusions since both coefficients have low $t$-statistics. They were nevertheless kept in the model to distinguish the effect of the trip purpose and the language region on choice.

- The positive sign of coefficient $\beta_{\text {comfort }}$ shows that a good perception of comfort of PT increases its utility. Let us recall that variable $P C P T$ was interacted with variable time $_{P T}$. When we look at the net effect of travel time in the utility of PT taking into account the interaction, we have:

$$
\beta_{\text {time }_{\mathrm{PT}}}+\frac{\beta_{\text {comfort }}}{1000} \cdot \text { PCPT. }
$$

Since $\beta_{\text {time }_{\text {PT }}}$ is negative and $\beta_{\text {comfort }}$ is positive, travel time sensitivity decreases with a better perception of comfort of PT. To make the time coefficient positive, the value of the LV should be 13.5 for the central evaluator. This value is very high considering the mean $\lambda_{\text {mean }}$, that is estimated as 3.33. A similar analysis can be done for the other evaluators. It can then be concluded that the net effect of travel time is hardly ever positive.

From the analysis of the estimated parameters of the structural equation of the LV PCPT we see that the coefficients differ more across the evaluators compared to the parameters of the choice model. 
- The negative sign of $\lambda_{\text {active }}$ indicates that individuals with full- or part-time jobs have a more negative perception of comfort of PT compared to those without jobs.

- Age is also a factor affecting the perception of comfort of PT, i.e. people below 50 years have a more negative image of it. We observe that the coefficient for age is not significant for the outlying evaluator.

- Living in a French-speaking region is negatively affecting the perception of comfort of PT.

- Finally, respondents with at least two cars in the household have a more negative image of comfort of PT compared to others.

The estimated parameters regarding the measurement equations of the indicators directly depend on the ratings given by the evaluators. Therefore the estimates of $\alpha_{k}, \gamma_{k}$ and $\sigma_{k}$ differ considerably with different set of ratings.

The estimation results of the logit model show that parameters $\lambda_{\text {French }}$ and $\lambda_{\text {age }_{50}}$ are not significant when they are directly included in the utility functions of the choice model. This can be due to the fact that some factors are correlated, namely French with French - time $e_{\mathrm{PT}}$, and age $_{50} \cdot$ time $_{\mathrm{PT}}$ with active time $_{\mathrm{PT}}$. The correlations are respectively 0.75 and 0.61 . This however indicates that the introduction of the perception of comfort of PT improves the explanatory power of the choice model. Socio-economic factors French and age $_{50}$ provide a better understanding of the model when integrated through an LVM rather than through a direct effect on the utility. 


\begin{tabular}{|c|c|c|c|c|c|c|c|c|c|c|c|c|}
\hline \multirow[b]{2}{*}{ Name } & \multicolumn{3}{|c|}{ Logit } & \multicolumn{3}{|c|}{ Central evaluator } & \multicolumn{3}{|c|}{ Outlying evaluator } & \multicolumn{3}{|c|}{ Median ratings } \\
\hline & Value & $t$-test & & Value & $t$-test & & Value & $t$-test & & Value & $t$-test & \\
\hline $\mathrm{ASC}_{\mathrm{PT}}$ & -0.178 & -0.88 & $*$ & -0.155 & -0.77 & $*$ & -0.132 & -0.66 & $*$ & -0.155 & -0.77 & $*$ \\
\hline $\mathrm{ASC}_{\mathrm{PMM}}$ & 0.423 & 2.30 & & 0.416 & 2.26 & & 0.410 & 2.22 & & 0.419 & 2.27 & \\
\hline$\beta_{\text {cost }}$ & -0.0658 & -8.67 & & -0.0637 & -8.11 & & -0.0628 & -8.02 & & -0.0653 & -8.08 & \\
\hline$\beta_{\text {timePT }}$ & -0.00600 & -3.34 & & -0.0143 & -7.71 & & -0.0293 & -4.17 & & -0.0208 & -7.06 & \\
\hline$\beta_{\text {timePMM }_{\text {PM }}}$ & -0.0330 & -10.27 & & -0.0313 & -9.53 & & -0.0312 & -9.55 & & -0.0323 & -9.43 & \\
\hline$\beta_{\text {distance }}$ & -0.236 & -11.51 & & -0.233 & -11.4 & & -0.233 & -11.38 & & -0.235 & -11.45 & \\
\hline$\beta_{\text {workPT }_{\text {PT }}}$ & 0.0987 & 0.42 & $*$ & -0.0602 & -0.26 & $*$ & -0.0928 & -0.40 & $*$ & -0.0474 & -0.20 & $*$ \\
\hline$\beta_{\text {workPMM }_{\text {PM }}}$ & -0.613 & -2.77 & & -0.572 & -2.58 & & -0.560 & -2.53 & & -0.575 & -2.60 & \\
\hline$\beta_{\text {FrenchPT }}$ & -0.228 & -0.61 & $*$ & -0.073 & -0.24 & $*$ & -0.113 & -0.37 & $*$ & -0.0808 & -0.26 & $*$ \\
\hline$\beta_{\text {French }_{\text {PMM }}}$ & 0.990 & 3.64 & & 0.966 & 3.56 & & 0.969 & 3.57 & & 0.967 & 3.56 & \\
\hline$\beta_{\text {comfort }}$ & - & - & & 1.06 & 3.46 & & 1.09 & 2.65 & & 1.33 & 4.34 & \\
\hline$\lambda_{\text {mean }}$ & - & - & & 3.33 & 9.40 & & 15.7 & 11.46 & & 7.47 & 9.98 & \\
\hline$\lambda_{\text {French }}$ & 1.11 & 0.44 & $*$ & -0.559 & -1.80 & & -0.139 & -0.48 & $*$ & -0.456 & -1.58 & $*$ \\
\hline$\lambda_{\text {age }_{50}}$ & 1.42 & 1.25 & $*$ & -1.30 & -5.53 & & 0.0643 & 0.30 & $*$ & -1.04 & -4.62 & \\
\hline$\lambda_{\text {active }}$ & -8.34 & -6.77 & & -1.10 & -4.37 & & -0.582 & -2.68 & & -1.12 & -4.62 & \\
\hline$\lambda_{\text {cars }}$ & -7.81 & -6.59 & & -0.730 & -3.06 & & -0.362 & -1.58 & $*$ & -0.688 & -3.04 & \\
\hline$\alpha_{2}$ & - & - & & -0.243 & -2.67 & & 0.505 & 1.61 & $*$ & 0.00209 & 0.01 & $*$ \\
\hline$\alpha_{3}$ & - & - & & -0.473 & -3.92 & & 1.46 & 2.91 & & -0.686 & -1.98 & \\
\hline$\alpha_{4}$ & - & - & & -1.14 & -9.33 & & -3.33 & -7.43 & & -2.34 & -6.55 & \\
\hline$\alpha_{5}$ & - & - & & -1.23 & -13.33 & & -0.344 & -0.90 & $*$ & -2.31 & -6.75 & \\
\hline$\alpha_{6}$ & - & - & & -1.22 & -11.7 & & 0.742 & 1.68 & & -2.98 & -7.49 & \\
\hline$\alpha_{7}$ & - & - & & -0.391 & -3.63 & & -2.30 & -5.51 & & -0.828 & -3.03 & \\
\hline$\alpha_{8}$ & - & - & & -0.560 & -5.03 & & 1.37 & 5.53 & & -1.35 & -3.97 & \\
\hline$\alpha_{9}$ & - & - & & -0.842 & -5.83 & & 0.731 & 2.49 & & -2.56 & -5.90 & \\
\hline$\gamma_{1}$ & - & - & & 0.255 & 10.55 & & 0.116 & 11.59 & & 0.230 & 9.00 & \\
\hline$\gamma_{2}$ & - & - & & 0.158 & 4.59 & & 0.0742 & 3.85 & & 0.151 & 3.94 & \\
\hline$\gamma_{3}$ & - & - & & 0.108 & 2.23 & & 0.00423 & 0.13 & $*$ & 0.203 & 4.18 & \\
\hline$\gamma_{4}$ & - & - & & 0.422 & 14.89 & & 0.319 & 30.33 & & 0.433 & 13.06 & \\
\hline$\gamma_{5}$ & - & - & & 0.195 & 5.69 & & 0.123 & 5.40 & & 0.321 & 7.49 & \\
\hline$\gamma_{6}$ & - & - & & 0.218 & 5.67 & & 0.051 & 1.77 & & 0.453 & 11.34 & \\
\hline$\gamma_{n}$ & - & - & & 0.344 & 12.71 & & 0.258 & 16.29 & & 0.324 & 10.36 & \\
\hline$\gamma_{8}$ & - & - & & 0.264 & 7.88 & & 0.0268 & 1.69 & & 0.336 & 8.62 & \\
\hline$\gamma_{9}$ & - & - & & 0.237 & 4.77 & & 0.0613 & 3.36 & & 0.448 & 10.38 & \\
\hline$\sigma_{1}$ & - & - & & -0.0585 & -1.17 & $*$ & -1.06 & -25.2 & & 0.0627 & 1.46 & $*$ \\
\hline$\sigma_{2}$ & - & - & & 0.255 & 6.09 & & -0.521 & -12.34 & & 0.388 & 9.85 & \\
\hline$\sigma_{3}$ & - & - & & 0.365 & 7.77 & & -0.365 & -8.29 & & 0.381 & 7.37 & \\
\hline$\sigma_{4}$ & - & - & & -0.344 & -2.81 & & -1.68 & -9.25 & & 0.00596 & 0.07 & $*$ \\
\hline$\sigma_{5}$ & - & - & & 0.0509 & 0.92 & $*$ & -0.461 & -7.56 & & 0.155 & 2.23 & \\
\hline$\sigma_{6}$ & - & - & & 0.00644 & 0.09 & $*$ & -0.394 & -7.64 & & -0.279 & -1.74 & $*$ \\
\hline$\sigma_{7}$ & - & - & & -0.250 & -2.92 & & -1.23 & -15.52 & & 0.0178 & 0.27 & $*$ \\
\hline$\sigma_{8}$ & - & - & & 0.122 & 1.99 & & -0.673 & -15.2 & & 0.270 & 4.39 & \\
\hline$\sigma_{9}$ & - & - & & 0.180 & 2.34 & & -0.675 & -11.56 & & 0.0496 & 0.48 & $*$ \\
\hline
\end{tabular}

Table 5: Estimation results for the logit model and the HCMs using the ratings of the central and outlying evaluators and the median ratings (sample size $=2^{\prime} 265$ ). 


\section{Validation}

From the estimation results presented in Section 4, we can conclude that the perception of comfort of PT has a significant impact on mode choice preferences. From Tables 10, 11 and 12 of the Appendix, we see that the associated parameter is indeed significant for all but one of the 25 evaluators (evaluator 25).

As a subsequent step we would like to assess the validation power of each model. In order to obtain indicators of validity of a statistical model, we compute the values of $\bar{\rho}^{2}$ and final loglikelihood of the choice model. The value of $\bar{\rho}^{2}$ is computed as follows.

$$
\bar{\rho}^{2}=1-\frac{\mathscr{L}(\hat{\beta})-J}{\mathscr{L}(0)}
$$

where $\mathscr{L}(\hat{\beta})$ is the final loglikelihood of the choice model component and $J$ is the number of parameters. The null loglikelihood $\mathscr{L}(0)$ is the likelihood of the models where all parameters are set to 0 .

The numerical values of loglikelihood and $\bar{\rho}^{2}$ are reported in Table 6 for the logit model and the DCM components of the three HCMs. We also show the number of parameters $J$ in each choice model component.

The loglikelihood and the values of $\bar{\rho}^{2}$ of the logit model are higher than those of the DCM components of the HCMs, implying a slightly better fit.

The fit indices of the model using the ratings of the outlying evaluator are slightly lower than the fit indices of the models using the ratings from the central evaluator and the median ratings. However the differences are small. A way to investigate how poor ratings affect the fit of the measurement model, compared to consistent ratings, is calculate the standardized residuals of the measurement equations. As an example, diagnostic plots of the standardized residuals for $I_{4}$ are shown for the outlying and central evaluators in Figure 4 of the Appendix. For the outlying evaluator, the standardized residuals for $I_{4}$ show a clear departure from normality.

\begin{tabular}{l|rrrr} 
Indicator & Logit & Central evaluator & Outlying evaluator & Median ratings \\
\hline Loglikelihood & -1153 & -1192 & -1199 & -1190 \\
$J$ & 14 & 11 & 11 & 11 \\
$\bar{\rho}^{2}$ & 0.443 & 0.425 & 0.422 & 0.427 \\
\hline
\end{tabular}

Table 6: Values of final loglikelihood and $\bar{\rho}^{2}$ for the logit model and the DCM components of the HCMs based on the ratings of the central and outlying evaluators and on the median ratings (sample size $=2^{\prime} 265$ ).

A thorough validation of the model would require its application on a different data set. As 
no other similar data set is available, we first estimate the models described in Section 3 on $80 \%$ of the data and in a second phase, we apply them on the remaining $20 \%$. The purpose of this procedure is to see how the fit indicators (final loglikelihood and value of $\bar{\rho}^{2}$ ) behave and how often the choice probabilities computed for each observation of the $20 \%$ of the data point to the actual choice. By choice probability, we mean the probability predicted by each model that the respondent chooses the transport mode he reported.

For comparison purposes, the logit model presented in Section 4 is also estimated on the same data set with $80 \%$ of the observations and validated on the data set with $20 \%$ of the observations.

Table 7 shows the loglikelihood, the number of parameters $J$, the value of $\bar{\rho}^{2}$ and the frequency of choice probabilities which are higher than 0.5 for the logit model and the three DCM components of the HCMs. All indices show that the prediction power is similar across models and confirm that it is rather high.

\begin{tabular}{l|r|rrr} 
Indicator & Logit & Central evaluator & Outlying evaluator & Median ratings \\
\hline Loglikelihood & -220 & -227 & -229 & -226 \\
$J$ & 14 & 11 & 11 & 11 \\
$\bar{\rho}^{2}$ & 0.459 & 0.449 & 0.444 & 0.452 \\
\hline Percentage choice probabilities $>0.5$ & $70.5 \%$ & $69.7 \%$ & $70.0 \%$ & $70.5 \%$ \\
\hline
\end{tabular}

Table 7: Values of final loglikelihood, $\bar{\rho}^{2}$ and proportion of choice probabilities higher than 0.5 for the logit model and the DCM components of the HCMs based on the ratings of the central and outlying evaluators and on the median ratings (sample size $=453$ ).

\section{Analysis of demand indicators across evaluators}

The estimation results of the mode choice models presented in Section 4 enable us to perform an analysis of demand for PT and PMM. In this section, we will precisely analyze how these indicators vary across models with ratings from different evaluators.

We compute three sorts of aggregate indicators: market shares, values of time (VOT) and elasticities.

\subsection{Definition of the indicators}

The indicators are computed as follows:

- The market share of an alternative $i$ is given by the weighted sum of the individual probabilities of choosing that particular alternative:

$$
M S(i)=\sum_{n=1}^{N} w_{n} P_{n}(i)
$$


where $w_{n}$ is the sample weight introduced in Section 2.1.

- The VOT is an indicator of willingness-to-pay (WTP), measuring the amount of money individuals are ready to spend if their trip can be reduced by one hour. For an individual $n$, the disaggregate VOT for a decrease of 1 hour in PMM is computed as follows:

$$
V O T_{\mathrm{PMM}, n}=\frac{\beta_{\text {time }_{\mathrm{PMM}}}}{\beta_{\text {cost }} \cdot 60}
$$

The disaggregate VOT for a decrease of 1 hour of travel in PT is given by the following formula:

$$
V O T_{\mathrm{PT}, n}=\frac{\beta_{\text {time }_{\mathrm{PT}}}+\beta_{\text {comfort }} \cdot \overline{\mathrm{PCPT}}_{n} / 1000}{\beta_{\text {cost }} \cdot 60},
$$

where $\overline{P C P T}_{n}$ is obtained by considering the mean of $P C P T$ of Equation (4). The aggregate VOTs for PMM and PT are obtained by computing the weighted sum of the above disaggregate indicators. Respectively, we have:

$$
\begin{gathered}
V O T_{\mathrm{PMM}}=\sum_{n=1}^{N} w_{n} V O T_{\mathrm{PMM}, n} \\
V O T_{\mathrm{PT}}=\sum_{n=1}^{N} w_{n} V O T_{\mathrm{PT}, n}
\end{gathered}
$$

- We report two types of aggregate elasticities, that is, direct elasticities and cross elasticities. We are interested in analyzing the percent change in the market shares of the alternatives of PMM, PT and SM, with respect to changes in variables cost $_{\mathrm{PMM}}$, cost $_{\mathrm{PT}}$, time $e_{\mathrm{PMM}}$ and time $_{\mathrm{PT}}$.

Aggregate direct elasticities are computed using the following formula, where $x_{i}$ is any of the above variables:

$$
E_{x_{i}}^{i}=\frac{\sum_{n=1}^{N} w_{n} P_{n}(i) E_{x_{i n}}^{i}}{\sum_{n=1}^{N} w_{n} P_{n}(i)} .
$$

In the above equation, $w_{n}$ is the sample weight of observation $n, P_{n}(i)$ is the probability that the individual who performed tour $n$ chooses alternative $i$ and $E_{x_{i n}}^{i}$ is the disaggregate direct elasticity of the demand for observation $n$ for variations in variable $x_{i n}$. This disaggregate elasticity is computed using the following expression:

$$
E_{x_{i n}}^{i}=\frac{\partial P_{n}(i)}{\partial x_{i n}} \frac{x_{i n}}{P_{n}(i)}
$$

To summarize, an aggregate direct elasticity denotes the percent change in the market share for alternative $i$ with respect to a change of $1 \%$ in the value of an attribute $x_{i}$ of $i$. 
Aggregate cross elasticities are given by the following expression:

$$
E_{x_{j}}^{i}=\frac{\sum_{n=1}^{N} w_{n} P_{n}(i) E_{x_{j n}}^{i}}{\sum_{n=1}^{N} w_{n} P_{n}(i)},
$$

where $E_{x_{j n}}^{i}$ is the cross elasticity of the demand for observation $n$ for variations in variable $x_{j n}$. This disaggregate cross elasticity is computed using the following expression:

$$
E_{x_{j n}}^{i}=\frac{\partial P_{n}(i)}{\partial x_{j n}} \frac{x_{j n}}{P_{n}(i)}
$$

An aggregate cross elasticity hence represents the percent change in the market share for alternative $i$ with respect to a change of $1 \%$ in the value of an attribute $x_{j}$ of another alternative $j$.

For the calculation of the elasticities, the partial derivatives are obtained by the built-in function of the software Biogeme. Note that the elasticity formula is not straightforward to derive, as time $e_{\mathrm{PT}}$ appears in two terms of the utility $V_{\mathrm{PT}}$.

\subsection{Aggregate indicators across evaluators}

To obtain a quantitative assessment of the demand for the transport modes, it is also interesting to look how indicators of demand differ across evaluators. For this purpose, the market shares, aggregate VOTs and elasticities for the models with different ratings of the evaluators are computed.

Market shares, VOTs and elasticities are presented in Table 13 in the Appendix. The results show that aggregate indicators of demand do not show important changes with different ratings of the adjectives. Small differences can be observed between the indicators computed for the logit model and the ones computed for the HCMs.

As expected the market share of PMM is rather high compared to PT. The use of SM is rather low. The VOTs show that individuals are willing to pay about $30 \mathrm{CHF}$ to gain one hour in a trip in PMM, whereas they are only ready to spend about 12 CHF to gain the same time in PT. We moreover note that these values are comparable with the ones reported in a study on Swiss values of travel time savings (Axhausen et al., 2008). We also conclude that the demand for the three types of transportation modes is rather inelastic with respect to changes in the travel fare or duration. The absolute value of the aggregate elasticities is indeed lower than 1 (Arnold, 2008). Nevertheless, the demand is more elastic with respect to changes in travel time than with respect to changes in travel cost. By considering the results of the central evaluator as an example, we can observe that an increase of $1 \%$ in the travel time in PT leads to a decrease of $0.62 \%$ of its market share and that the same percent increase in the time in PMM leads to an increase of $0.61 \%$ in the market share of PT. Similar conclusions can be drawn for the other evaluators. 


\subsection{Disaggregate indicators across evaluators}

After investigating the market shares, VOTs and elasticities of cost or time for each evaluator, we are interested in analyzing the distribution of such indicators over all evaluators. In particular, we want to analyze the distribution of (i) disaggregate demand indicators for three respondents of the initial RP survey and (ii) aggregate demand indicators computed over the whole sample.

To analyze the distributions of the disaggregate demand indicators over all evaluators, we select three examples of respondents: a respondent whose probability to select the alternative he actually chose (PT) is poorly predicted by the model, a respondent with an average predicted probability $(\cong 0.5)$ for the chosen alternative $(\mathrm{PT})$ and a respondent whose predicted probability to select the chosen alternative (PMM) is high. Means and standard deviations (SD) for these disaggregate demand indicators are computed over the sample of evaluators and reported in columns 'Low', 'Medium' and 'High' of Table 8. Aggregate demand indicators are also obtained from the application of each HCM on the sample of all 2'265 respondents of the RP survey. Their means and SDs over all evaluators are reported in column 'All observations' of Table 8.

From Table 8 we observe a general trend: the distribution of disaggregate indicators across evaluators has a larger variance than the distribution of aggregate indicators. This holds for every indicator, except VOT.

For the three chosen respondents, the probability of choosing an alternative has a larger standard deviation than the market share of the same alternative. Figure 1 provides a visual example of this phenomenon for the probability of choosing the PT alternative. For each of the four graphs, we considered the same scale for the horizontal axes, in order to make the comparison between the four distributions easier ${ }^{2}$. We mean that the difference between the extreme points of the axis is the same across graphs.

Similarly, Figure 2 shows that the disaggregate elasticities of the cost of PMM are more spread than the aggregate cost elasticities.

The aggregate VOT for PT has a standard deviation which is smaller than the disaggregate VOTs for PT for two out of the three examples of respondents (see Table 8 and Figure 3). In addition, we have a different VOT for PT for each individual due to the interaction with the perception of comfort of PT, while the VOT for PMM is constant across respondents.

\subsection{Impact of an increase of the comfort level}

We present here an example demonstrating an important property of HCMs, that is, we can measure in a quantitative way the impact of a change in the perceptional variable on the choice. In

\footnotetext{
${ }^{2}$ The same remark holds for Figures 2 and 3 .
} 


\begin{tabular}{|c|c|c|c|c|c|c|c|c|c|}
\hline \multirow{3}{*}{ Indicator } & \multirow{3}{*}{ Mode } & \multicolumn{6}{|c|}{$\begin{array}{l}\text { Examples of respondents with different probabilities for } \\
\text { the chosen alternative }\end{array}$} & \multicolumn{2}{|c|}{ All observations } \\
\hline & & \multicolumn{2}{|c|}{ Low } & \multicolumn{2}{|c|}{ Medium } & \multicolumn{2}{|c|}{ High } & \multirow[b]{2}{*}{ Mean } & \multirow[b]{2}{*}{ SD } \\
\hline & & Mean & SD & Mean & SD & Mean & SD & & \\
\hline \multirow{3}{*}{$\begin{array}{l}\text { Probability of choice / } \\
\text { Market share }\end{array}$} & PT & 0.047 & 0.008 & 0.543 & 0.013 & 0.067 & 0.005 & 0.278 & 0.001 \\
\hline & PMM & 0.953 & 0.008 & 0.457 & 0.013 & 0.933 & 0.005 & 0.659 & 0.001 \\
\hline & SM & 0.000 & 0.000 & 0.000 & 0.000 & 0.000 & 0.000 & 0.063 & 0.000 \\
\hline \multirow{2}{*}{ VOT } & PT & 10.417 & 0.603 & 11.617 & 0.320 & 13.584 & 0.538 & 12.311 & 0.323 \\
\hline & PMM & 29.635 & 0.108 & 29.635 & 0.108 & 29.635 & 0.108 & 29.635 & 0.108 \\
\hline \multirow{2}{*}{ Elasticity of cost } & PT & -2.681 & 0.036 & -0.247 & 0.008 & -1.493 & 0.022 & -0.282 & 0.001 \\
\hline & PMM & -0.026 & 0.005 & -0.246 & 0.006 & -0.036 & 0.002 & -0.073 & 0.000 \\
\hline \multirow{2}{*}{ Elasticity of time } & PT & -2.209 & 0.379 & -0.914 & 0.046 & -2.122 & 0.039 & -0.626 & 0.005 \\
\hline & PMM & -0.120 & 0.021 & -1.239 & 0.027 & -0.097 & 0.007 & -0.276 & 0.001 \\
\hline \multirow{2}{*}{$\begin{array}{l}\text { Cross-elasticity of cost } \\
\text { of PMM }\end{array}$} & SM & 0.562 & 0.005 & 0.241 & 0.005 & 0.512 & 0.011 & 0.040 & 0.001 \\
\hline & $\mathrm{PT}$ & 0.531 & 0.007 & 0.207 & 0.007 & 0.499 & 0.007 & 0.163 & 0.000 \\
\hline \multirow{2}{*}{$\begin{array}{l}\text { Cross-elasticity of cost } \\
\text { of PT }\end{array}$} & PMM & 0.131 & 0.023 & 0.293 & 0.007 & 0.107 & 0.007 & 0.115 & 0.001 \\
\hline & SM & 0.131 & 0.023 & 0.293 & 0.007 & 0.107 & 0.007 & 0.048 & 0.001 \\
\hline \multirow{2}{*}{$\begin{array}{l}\text { Cross-elasticity of time } \\
\text { in PMM }\end{array}$} & SM & 2.591 & 0.024 & 1.216 & 0.027 & 1.402 & 0.029 & 0.188 & 0.003 \\
\hline & PT & 2.446 & 0.032 & 1.044 & 0.034 & 1.365 & 0.020 & 0.610 & 0.002 \\
\hline \multirow{2}{*}{$\begin{array}{l}\text { Cross-elasticity of time } \\
\text { in PT }\end{array}$} & PMM & 0.105 & 0.008 & 1.084 & 0.008 & 0.151 & 0.011 & 0.254 & 0.002 \\
\hline & SM & 0.105 & 0.008 & 1.084 & 0.008 & 0.151 & 0.011 & 0.110 & 0.001 \\
\hline
\end{tabular}

Table 8: Probabilities of choice, disaggregate VOTs, direct and cross-elasticities for three respondents of the RP survey (Mean and SD over all 25 evaluators); market shares, aggregate VOTs, direct and crosselasticities resulting from the application of each HCM on the sample of all $2^{\prime} 265$ respondents of the RP survey (Mean and SD over all 25 evaluators).

the present case study, we can indeed quantify the effect of an increase in the comfort level on the mode choice. By construction, changes in the variables characterizing a certain level of comfort of PT lead to an increase or decrease of the perception of comfort of PT, which results in variations in the market shares.

The scenario presented in this section is based on the estimation sample, where the number of cars in each household has been decreased by 1 . Table 9 shows that the market share of PT has slightly increased as a consequence of this potential policy change. A better perception of comfort of PT can hence drive individuals to make an increased use of such transportation modes.

\section{Some recommendations for questionnaire design}

The outcomes of this research have shown that more qualitative data such as adjectives can be used to measure a perception. However the adjective data set we are using was not initially designed 

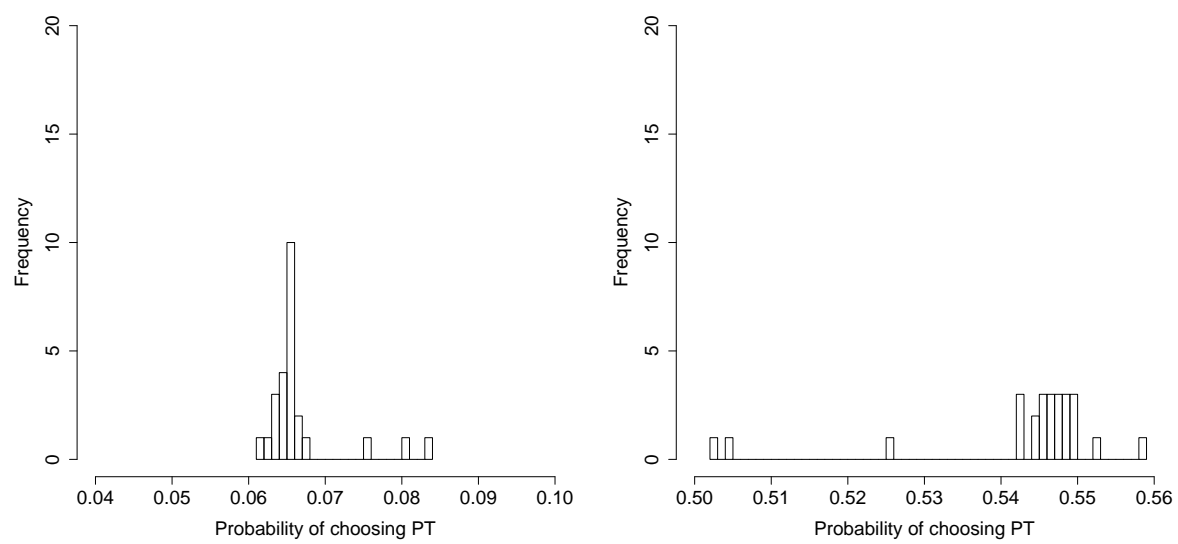

(a) Respondent with a high probability for the (b) Respondent with an average probability for chosen alternative (PMM) the chosen alternative (PT)

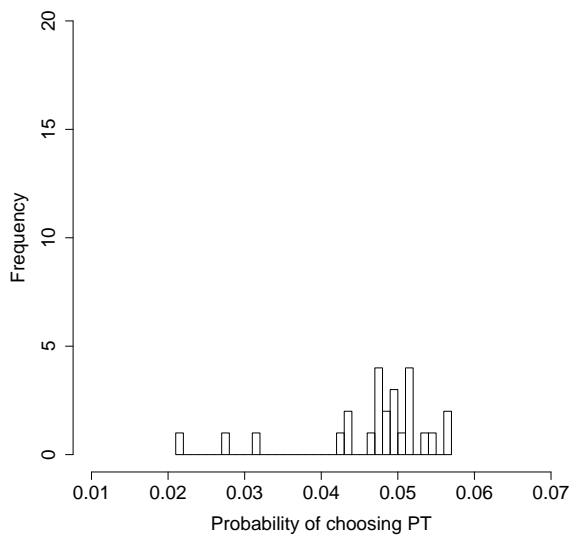

(c) Respondent with a low probability for the chosen alternative (PT)

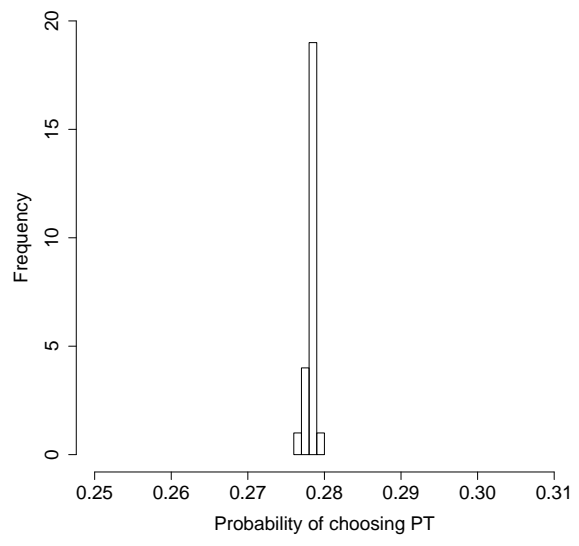

(d) Aggregation over all respondents

Figure 1: Histograms of the probabilities of choosing PT for three respondents and histogram of the market share of PT (sample size $=25$ ).

to be used for quantitative research. The whole data collection procedure could therefore benefit from some improvements in order to be faster and comparable with existing approaches. In this section, we report some recommendations for future survey design.

The two surveys in one step. The RP data collection and the collection of ratings of the adjectives could be performed in a single step, where the respondents of the RP survey would be asked to evaluate the adjectives. The detailed survey methodology would consist of (1) asking them to report freely adjectives for the different transportation modes, (2) asking them to which theme they associate which reported adjectives and (3) rate each adjective on the scale of the given theme. 

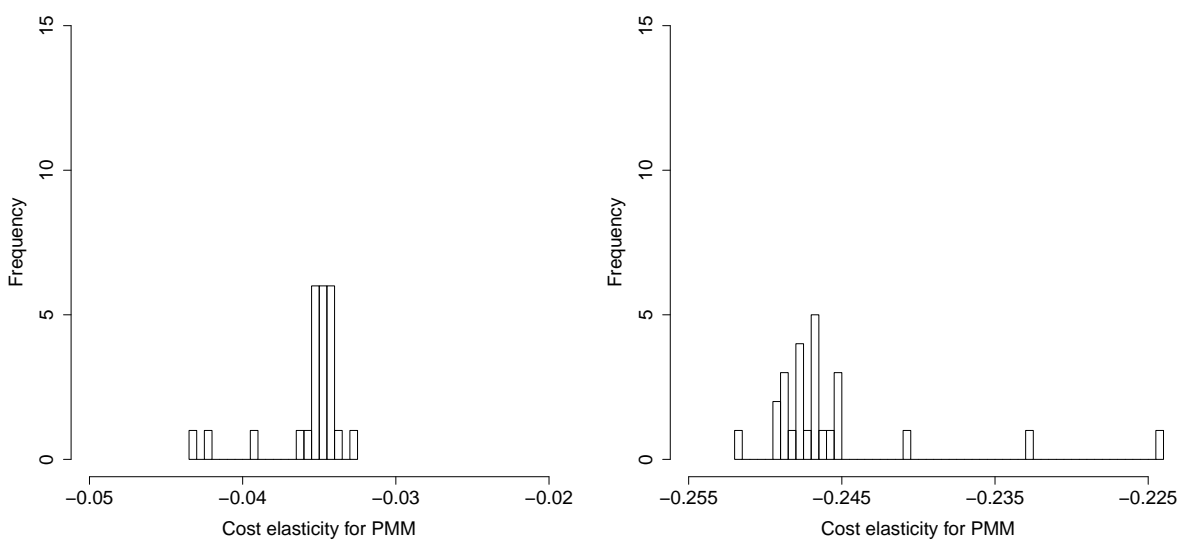

(a) Respondent with a high probability for the (b) Respondent with an average probability for chosen alternative (PMM) the chosen alternative (PT)

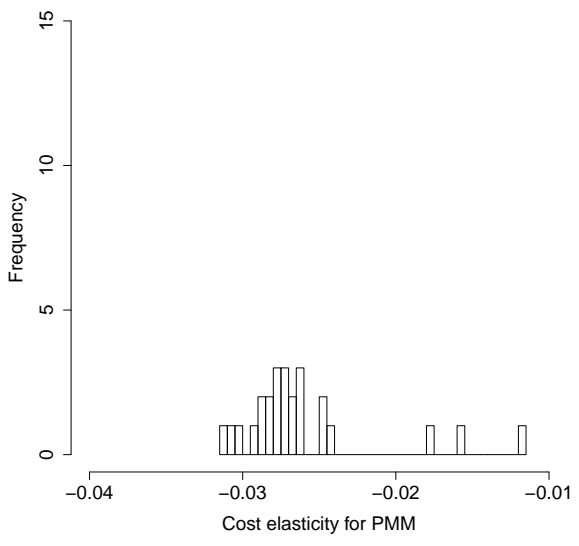

(c) Respondent with a low probability for the chosen alternative (PT)

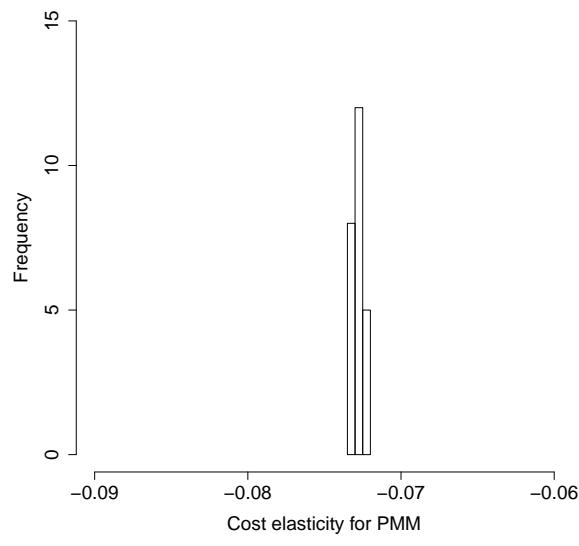

(d) Aggregation over all respondents

Figure 2: Histograms of the disaggregate cost elasticities for PMM for three respondents and histogram of the aggregate cost elasticities for PMM (sample size $=25$ ).

A comparative approach with the classical method. A standard way to measure perceptions is to ask respondents to rate their agreement to opinion statements on a five-point Likert scale. As highlighted earlier, such a method depends on the conception of the perception by the survey designer. However, as raised by a reviewer, the methodology presented in this paper also involves some subjectivity, in the sense that the adjectives are associated to a theme by an analyst.

Since multiple evaluators are involved, the bias linked to the adjective rating is reduced, but it could be argued that in the case of the design of opinion statements, some bias is also reduced since several individuals are usually involved in the survey design. 

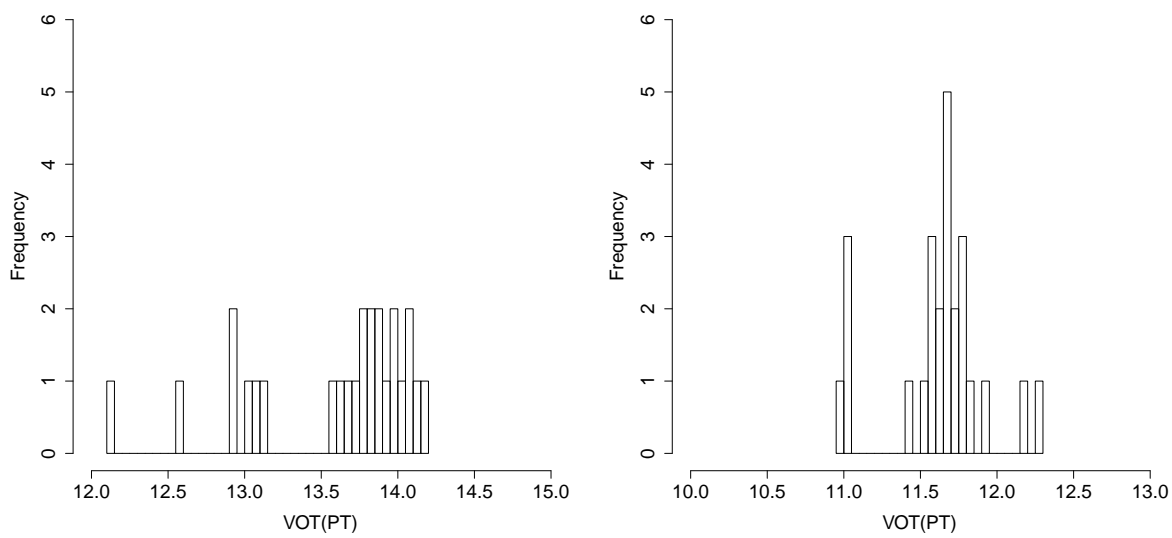

(a) Respondent with a high probability for the (b) Respondent with an average probability for chosen alternative (PMM) the chosen alternative (PT)

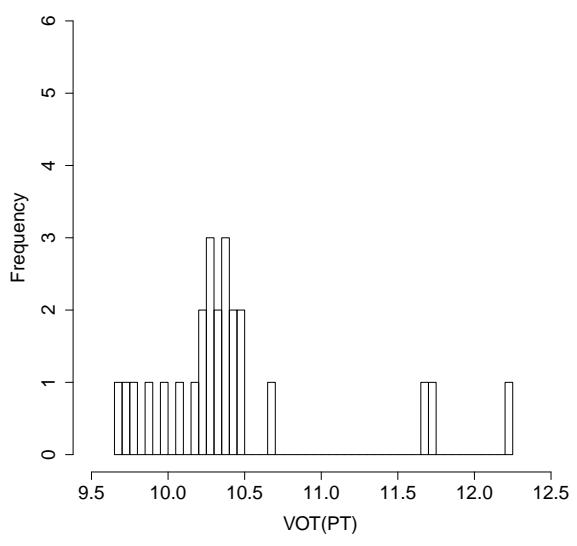

(c) Respondent with a low probability for the chosen alternative (PT)

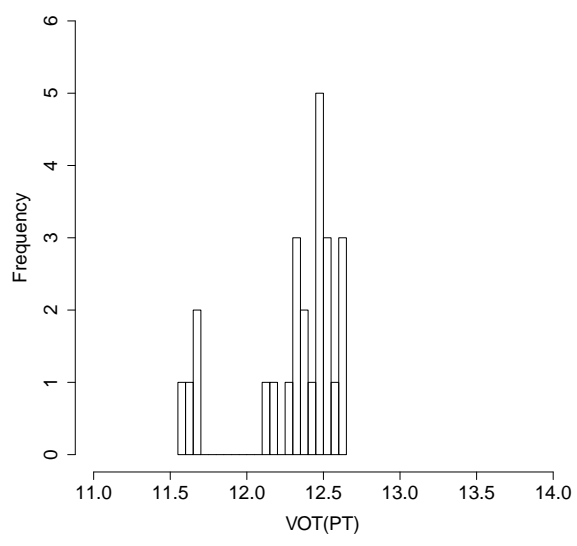

(d) Aggregation over all respondents

Figure 3: Histograms of the VOTs for three respondents for PT and histogram of the aggregate VOT for PT (sample size $=25$ ).

Therefore, an interesting research path to investigate would be to separate the RP respondents into two groups and ask the first one to answer opinion statements related to a number of themes of interest (e.g. including comfort) and the second one to report adjectives related to the same themes.

\section{Conclusion and further works}

This research presents a methodology to measure and integrate perceptional information into a DCM. The use of semi-open questions provides spontaneous information about respondents' perceptions, and the estimation and application of an HCM have shown that the latter affect the choice in a significant way. 


\begin{tabular}{ll|rr|lr}
\multirow{2}{*}{ Indicator } & Mode & \multicolumn{2}{|c|}{ Base case } & \multicolumn{2}{|c}{ Less cars per households } \\
& & Mean & SD & Mean & SD \\
\hline \multirow{3}{*}{ Market share } & PT & 0.278 & 0.001 & 0.283 & 0.002 \\
& PMM & 0.659 & 0.001 & 0.654 & 0.002 \\
& SM & 0.063 & 0.000 & 0.062 & 0.000 \\
\hline
\end{tabular}

Table 9: Market shares for the whole sample of the RP survey under a scenario where the number of cars per household is decreased (sample size $=2^{\prime} 265$ ).

This paper contributes to several achievements. First, to the best of our knowledge, this is the first attempt to integrate qualitative information, such as semi-open questions, into DCMs. We achieve a meaningful characterization of the perception of comfort of public transportation by using the adjectives as measurements of it. Second, we provide a methodology to rate the adjectives on a scale of comfort which minimizes the subjectivity resulting when using a single modeler's ratings. Third, we highlight the impact of ratings from different evaluators on the demand by exploring several indicators, such as market shares, values of time and elasticities.

By considering responses to semi-open questions, we aim at proposing an alternative way to measure perceptions. The obtained adjectives contain rich information about the individuals' perceptions since they are freely reported. This way we wish to overcome the bias inherent to the collection of responses to opinion statements, which are reflecting the survey designer's representation of a perception. The methodology moreover highlights the importance of considering mixed qualitative/quantitative approach to conduct revealed or stated preferences surveys.

Future works involve the integration of the ratings of all evaluators into the HCM framework, in order to benefit from the global judgement to valuate adjectives. In addition, we will also include ratings from a finer-grained scale, ranging from -1000 to 1000 , which were reported by additional evaluators. Since differences across evaluators were observed in disaggregate indicators, a finer characterization of perceptions will be investigated as a future research. For this purpose we plan to integrate the socio-economic information of the evaluators as explanatory variables of the reported ratings.

The effect of the perception of comfort of other transport modes might also affect the choices of individuals. Moreover its interaction with other variables could be tested, since the perception of comfort might differ among population segments. The list of reported adjectives revealed eleven themes (see Section 2.2) which might also be important factors of transportation mode choices. In future research, all these aspects should be investigated, in order to have a broader view of all perceptional variables affecting transportation mode decisions. However, it is important to remark 
that in practice, rating adjectives relative to eleven themes can be burdensome for evaluators. Such an exercice should be designed to minimize the impact of the fatigue on the quality of the evaluation. Clearly, using many evaluators would ease the burden for each of them. In addition, it is worth noting that the presented modeling methodology shares the same limitations with HCMs. First, when the number of indicators for the latent construct is high, the maximum likelihood estimation becomes cumbersome, and other estimation techniques (e.g. Bayesian) should be considered. Second, more work needs to be done to validate how well the structural equation is able to characterize an LV. In particular, a good measure of the fit would be useful to support this analysis.

Regarding survey design, further work is needed to assess whether all the measures of a psychological construct which is an important explanatory factor of a respondent's behavior are actually recalled. The understanding of this cognitive process will allow to highlight better the strengths and weaknesses of semi-open questions versus opinion statements as measurements of a perception.

In this paper, we have presented a method that exploits a new type of data, and illustrated it on a real case study. Note that the additional complexity can be a curse or a blessing depending on the application, the data and the context in general. For example, the use of semi-open questions for similar concepts (like 'comfort' and 'convenience') may complicate the task of the evaluators, as the same adjectives may be reported for both concepts. The associated modeling issues are yet to be investigated.

\section{Acknowlegdements}

The authors would like to thank PostBus for their advice and feedback on the collaborative project which was set up and for funding it. They also would like to acknowledge the Urban Sociology Laboratory (LaSUR) and the Urban and Regional Planning Laboratory (CEAT) who co-conducted the qualitative and the RP surveys which serve as a basis to this research. They are also grateful to Alberto Monticone, who established the classification of the adjectives into themes. This paper has also benefited from the comments from three anonymous reviewers as well as two anonymous reviewers of an earlier version (Glerum et al., 2011) which was part of the conference proceedings of the ICMC conference in Leeds in 2011.

\section{References}

Abou-Zeid, M., Ben-Akiva, M., Bierlaire, M., Choudhury, C. and Hess, S. (2010). Attitudes and value of time heterogeneity, in E. V. de Voorde and T. Vanelslander (eds), Applied Transport 
Economics A Management and Policy Perspective, de boeck, pp. 523-545.

Abou-Zeid, M., Witter, R., Bierlaire, M., Kaufmann, V. and Ben-Akiva, M. (2012). Happiness and travel mode switching: Findings from a swiss public transportation experiment, Transport Policy 19(1): 93-104.

Amazon's Mechanical Turk (2005-2012).

URL: https://www.mturk.com/

Arnold, R. A. (2008). Economics, ninth edn, Cengage Learning, Mason.

Atasoy, B., Glerum, A. and Bierlaire, M. (2013). Attitudes towards mode choice in Switzerland, disP - The Planning Review 49(2): 101-117.

Axhausen, K. W., Hess, S., König, A., Abay, G., Bates, J. J. and Bierlaire, M. (2008). Income and distance elasticities of values of travel time savings: New Swiss results, Transport Policy 15(3): 173-185.

Banerjee, M., Capozzoli, M., McSweeney, L. and Sinha, D. (1999). Beyond kappa: A review of interrater agreement measures, The Canadian Journal of Statistics 27(1): 3-23.

Bearden, W. and Netemeyer, R. (1999). Handbook of marketing scales: multi-item measures for marketing and consumer behavior research, Association for Consumer Research, Sage Publications.

Beirao, G. and Cabral, J. S. (2007). Understanding attitudes towards public transport and private car: A qualitative study, Transport Policy 14(6): 478-489.

Ben-Akiva, M. and Lerman, S. (1985). Discrete choice analysis: theory and application to travel demand, Vol. 9, The MIT Press.

Ben-Akiva, M., McFadden, D., Train, K., Walker, J., Bhat, C., Bierlaire, M., Bolduc, D., BoerschSupan, A., Brownstone, D., Bunch, D., Daly, A., de Palma, A., Gopinath, D., Karlstrom, A. and Munizaga, M. A. (2002). Hybrid choice models: Progress and challenges, Marketing Letters 13(3): 163-175.

Bierlaire, M., Curchod, A., Danalet, A., Doyen, E., Faure, P., Glerum, A., Kaufmann, V., Tabaka, K. and Schuler, M. (2011). Projet de recherche sur la mobilité combinée, rapport définitif de l'enquête de préférences révélées, Technical Report TRANSP-OR 110704, Transport and Mobility Laboratory, Ecole Polytechnique Fédérale de Lausanne. 
Bierlaire, M. and Fetiarison, M. (2009). Estimation of discrete choice models: extending BIOGEME, Swiss Transport Research Conference (STRC).

Bolduc, D. and Daziano, R. A. (2011). Incorporating pro-environmental preferences toward green automobile technologies through a Bayesian Hybrid Choice Model, Transportmetrica pp. 1-33.

Brownstone, D. (1998). Multiple imputation methodology for missing data, non-random response, and panel attrition, Theoretical foundations of travel choice modelling, Elsevier, New York, Amsterdam, pp. 421-450.

Cramer, P. (1968). Word association, Academic Press.

Daly, A., Hess, S., Patruni, B., Potoglou, D. and Rohr, C. (2012). Using ordered attitudinal indicators in a latent variable choice model: a study of the impact of security on rail travel behaviour, Transportation 39: 267-297.

Espino, R., Román, C. and Ortúzar, J. d. D. (2006). Analyzing demand for suburban trips: A mixed RP/SP model with latent variables and interaction effects, Transportation 33(3): 241-261.

Franklin, M. J., Kossmann, D., Kraska, T., Ramesh, S. and Xin, R. (2011). Crowddb: answering queries with crowdsourcing, Proceedings of the 2011 ACM SIGMOD International Conference on Management of data, SIGMOD '11, ACM, New York, NY, USA, pp. 61-72.

Glerum, A., Atasoy, B., Monticone, A. and Bierlaire, M. (2011). Adjectives qualifying individuals' perceptions impacting on transport mode preferences, Proceedings of the Second International Choice Modeling Conference, Leeds, UK.

Greene, W. H. and Hensher, D. A. (2003). A latent class model for discrete choice analysis: contrasts with mixed logit, Transportation Research Part B: Methodological 37(8): 681-698.

Hess, S. and Train, K. (2011). Recovery of inter- and intra-personal heterogeneity using mixed logit models, Transportation Research Part B: Methodological 45(7): 973-990.

Kaufmann, V., Jemelin, C. and Guidez, J.-M. (2001). Automobile et modes de vie urbains quel degré de liberté?, Documentation Française.

Kaufmann, V., Tabaka, K., Louvet, N. and Guidez, J.-M. (2010). Et si les Français n'avaient plus seulement une voiture dans la tête?, Certu.

Likert, R. (1932). A technique for the measurement of attitudes, Archives of Psychology 22(140). 
Looker, E., Denton, M. A. and Davis, C. K. (1989). Bridging the gap: Incorporating qualitative data into quantitative analyses, Social Science Research 18(4): 313-330.

McFadden, D. and Train, K. (2000). Mixed MNL models for discrete response, Journal of Applied Econometrics 15(5): 447-470.

Mossholder, K. W., Settoon, R. P., Harris, S. G. and Armenakis, A. A. (1995). Measuring emotion in open-ended survey responses: An application of textual data analysis, Journal of Management 21(2): 335-355.

Potkay, C. R. and Allen, B. P. (1973). The adjective generation technique: an alternative to adjective check lists, Psychological Reports 32: 457-458.

Robin, T., Bierlaire, M. and Cruz, J. (2011). Dynamic facial expression recognition with a discrete choice model, Journal of Choice Modelling 4(2): 95-148.

Schuman, H. and Presser, S. (1996). Questions and answers in attitude surveys: experiments on question form, wording, and context, Quantitative Studies in Social Relations, Sage Publications.

Snow, R., O'Connor, B., Jurafsky, D. and Ng, A. Y. (2008). Cheap and fast-but is it good?: evaluating non-expert annotations for natural language tasks, Proceedings of the Conference on Empirical Methods in Natural Language Processing, EMNLP '08, Association for Computational Linguistics, Stroudsburg, PA, USA, pp. 254-263.

Sorci, M., Antonini, G., Cruz, J., Robin, T., Bierlaire, M. and Thiran, J.-P. (2010). Modelling human perception of static facial expressions, Image and Vision Computing 28(5): 790-806.

Thorndike, E. L. (1920). A constant error in psychological ratings, Journal of Applied Psychology 4: $469-477$.

Venetis, P., Garcia-Molina, H., Huang, K. and Polyzotis, N. (2012). Max algorithms in crowdsourcing environments, Proceedings of the 21st international conference on World Wide Web, WWW '12, ACM, New York, NY, USA, pp. 989-998.

Vredin Johansson, M., Heldt, T. and Johansson, P. (2006). The effects of attitudes and personality traits on mode choice, Transportation Research Part A: Policy and Practice 40(6): 507-525.

Walker, J. and Ben-Akiva, M. (2002). Generalized random utility model, Mathematical Social Sciences 43(3): 303-343. 
Walker, J. L. (2001). Extended Discrete Choice Models: Integrated Framework, Flexible Error Structures, and Latent Variables, PhD thesis, Massachusetts Institute of Technology.

Yáñez, M., Raveau, S. and Ortúzar, J. d. D. (2010). Inclusion of latent variables in mixed logit models: Modelling and forecasting, Transportation Research Part A: Policy and Practice 44(9): 744-753.

\section{Appendix}
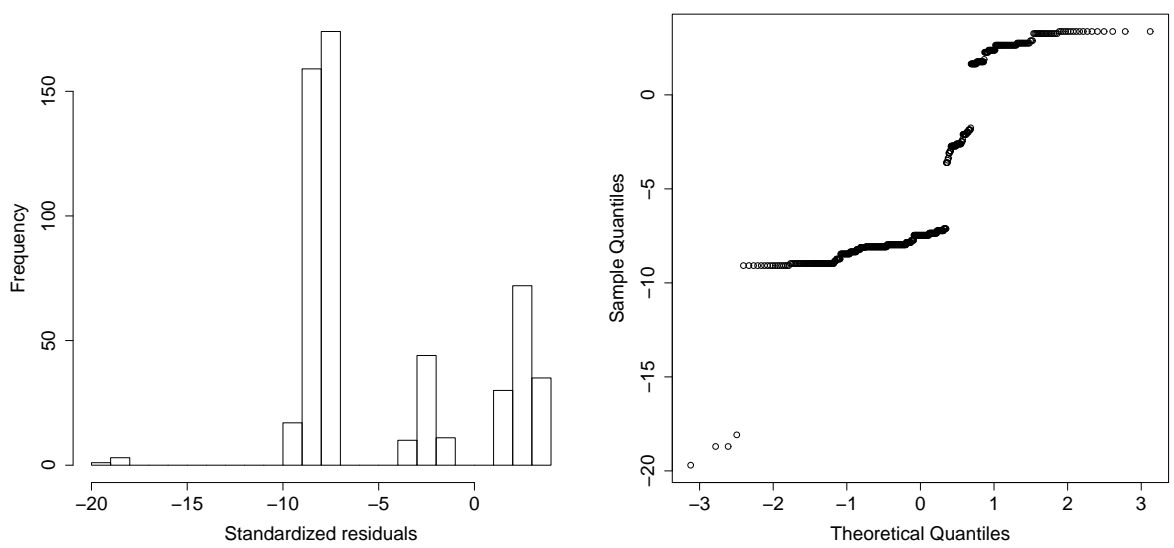

(a) Histogram of standardized residuals (Outly- (b) Normal Q-Q of standardized residuals ing evaluator) (Outlying evaluator)
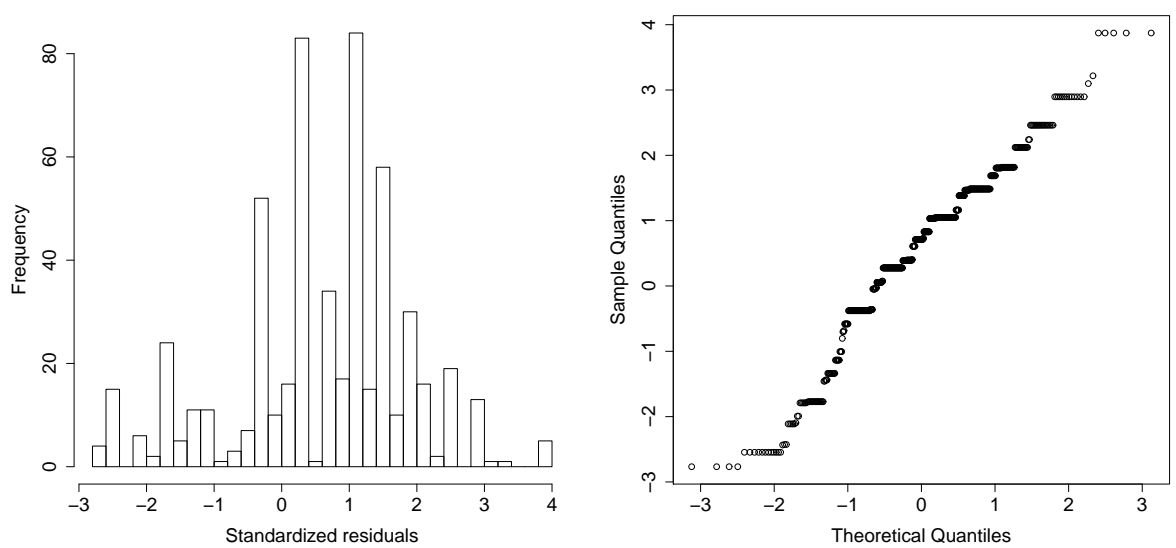

(c) Histogram of standardized residuals (Cent- (d) Normal Q-Q of standardized residuals ral evaluator) (Central evaluator)

Figure 4: Diagnostic plots of the standardized residuals for measurement equation relative to $I_{4}$ (sample size $\left.=2^{\prime} 265\right)$. 


\begin{tabular}{|c|c|c|c|c|c|c|c|c|c|c|c|c|c|c|c|c|c|c|c|c|c|c|}
\hline \multirow[b]{2}{*}{ Name } & \multicolumn{2}{|c|}{ Evaluator 1} & \multicolumn{2}{|c|}{ Evaluator 2} & \multicolumn{2}{|c|}{ Evaluator 3} & \multicolumn{2}{|c|}{ Evaluator 4} & \multicolumn{2}{|c|}{ Evaluator 5} & \multicolumn{2}{|c|}{ Evaluator 6} & \multicolumn{2}{|c|}{$\begin{array}{c}\text { Evaluator } 7 \\
\text { (central evaluator) }\end{array}$} & \multicolumn{2}{|c|}{ Evaluator 8} & \multicolumn{2}{|c|}{$\begin{array}{c}\text { Evaluator } 9 \\
\text { (outyling evaluator) } \\
\end{array}$} & \multicolumn{2}{|c|}{ Evaluator 10} & \multicolumn{2}{|c|}{ Evaluator 11} \\
\hline & Value & t-test & Value & t-test & Value & t-test & Value & t-test & Value & t-test & Value & t-test & Value & t-test & Value & t-test & Value & t-test & Value & t-test & Value & t-test \\
\hline $\mathrm{ASC}_{\mathrm{PT}}$ & -0.152 & -0.76 & -0.145 & -0.72 & -0.159 & -0.79 & -0.165 & -0.82 & -0.151 & -0.75 & -0.168 & -0.83 & -0.155 & -0.77 & -0.161 & -0.8 & -0.132 & -0.66 & -0.157 & -0.78 & -0.148 & -0.74 \\
\hline $\mathrm{ASC}_{\mathrm{PMM}}$ & 0.415 & 2.25 & 0.415 & 2.25 & 0.419 & 2.28 & 0.421 & 2.28 & 0.415 & 2.25 & 0.422 & 2.29 & 0.416 & 2.26 & 0.419 & 2.27 & 0.41 & 2.22 & 0.419 & 2.27 & 0.417 & 2.26 \\
\hline$\beta_{\text {cost }}$ & -0.0644 & -8.05 & -0.065 & -8.16 & -0.0648 & -8.08 & -0.0651 & -8.07 & -0.0644 & -8.06 & -0.0648 & -8.15 & -0.0637 & -8.11 & -0.0649 & -8.07 & -0.0628 & -8.02 & -0.0653 & -8.14 & -0.0653 & -8.15 \\
\hline$\beta_{\text {time }_{\mathrm{PT}}}$ & -0.0156 & -7.49 & -0.0181 & -7.11 & -0.0199 & -7.07 & -0.0183 & -7.45 & -0.0156 & -7.47 & -0.0164 & -7.79 & -0.0143 & -7.71 & -0.0182 & -7.44 & -0.0293 & -4.17 & -0.0179 & -7.46 & -0.0193 & -6.87 \\
\hline$\beta_{\text {timepMM }}$ & -0.0316 & -9.45 & -0.0319 & -9.48 & -0.0321 & -9.46 & -0.0323 & -9.45 & -0.0317 & -9.45 & -0.032 & -9.5 & -0.0313 & -9.53 & -0.0321 & -9.46 & -0.0312 & -9.55 & -0.0321 & -9.47 & -0.0321 & -9.46 \\
\hline$\beta_{\text {distance }}$ & -0.234 & -11.41 & -0.234 & -11.43 & -0.234 & -11.43 & -0.235 & -11.44 & -0.234 & -11.41 & -0.234 & -11.43 & -0.233 & -11.4 & -0.235 & -11.43 & -0.233 & -11.38 & -0.235 & -11.44 & -0.235 & -11.44 \\
\hline$\beta_{\text {workPT }_{\text {PT }}}$ & -0.0555 & -0.24 & -0.0618 & -0.26 & -0.0492 & -0.21 & -0.0399 & -0.17 & -0.0555 & -0.24 & -0.0355 & -0.15 & -0.0602 & -0.26 & -0.0476 & -0.2 & -0.0928 & -0.4 & -0.0487 & -0.21 & -0.0552 & -0.24 \\
\hline$\beta_{\text {time }_{\text {PMM }}}$ & -0.573 & -2.59 & -0.571 & -2.58 & -0.575 & -2.6 & -0.577 & -2.6 & -0.573 & -2.59 & -0.576 & -2.6 & -0.572 & -2.58 & -0.575 & -2.6 & -0.56 & -2.53 & -0.574 & -2.59 & -0.573 & -2.59 \\
\hline$\beta_{\text {French }_{\mathrm{PT}}}$ & -0.0855 & -0.28 & -0.133 & -0.43 & -0.0428 & -0.14 & -0.0088 & -0.03 & -0.0897 & -0.29 & -0.0067 & -0.02 & -0.073 & -0.24 & -0.035 & -0.11 & -0.113 & -0.37 & -0.0736 & -0.24 & -0.114 & -0.37 \\
\hline$\beta_{\text {French }_{\mathrm{PMM}}}$ & 0.968 & 3.56 & 0.972 & 3.58 & 0.963 & 3.55 & 0.961 & 3.54 & 0.968 & 3.57 & 0.96 & 3.53 & 0.966 & 3.56 & 0.963 & 3.55 & 0.969 & 3.57 & 0.966 & 3.56 & 0.97 & 3.57 \\
\hline$\beta_{\text {comfort }}$ & $-1.21^{* *}$ & -3.69 & $-1.21^{* *}$ & -3.89 & 1.27 & 4.1 & 1.36 & 4.3 & $-1.2^{* * *}$ & -3.67 & 1.29 & 4.3 & 1.06 & 3.46 & 1.29 & 4.21 & 1.09 & 2.65 & 1.29 & 4.25 & $-1.25^{* *}$ & -4.05 \\
\hline$\lambda_{\text {mean }}$ & -3.97 & -8.93 & -5.71 & -8.59 & 7.14 & 10.64 & 5.7 & 10.64 & -4.01 & -8.82 & 4.73 & 11.28 & 3.33 & 9.4 & 5.78 & 10.53 & 15.7 & 11.46 & 5.56 & 9.84 & -6.62 & -7.79 \\
\hline$\lambda_{\text {French }}$ & 0.447 & 1.41 & -0.0646 & -0.22 & -0.83 & -2.83 & -1.12 & -3.58 & 0.401 & 1.26 & -1.14 & -3.64 & -0.559 & -1.8 & -0.89 & -3.02 & -0.139 & -0.48 & -0.512 & -1.76 & 0.143 & 0.47 \\
\hline$\lambda_{\mathrm{age}_{50}}$ & 1.04 & 4.34 & 0.984 & 4.31 & -1.07 & -4.71 & -1.09 & -4.59 & 1.06 & 4.41 & -1.5 & -6.29 & -1.3 & -5.53 & -1.03 & -4.52 & 0.0643 & 0.3 & -1.15 & -5.03 & 1.09 & 4.68 \\
\hline$\lambda_{\text {active }}$ & 1.18 & 4.59 & 0.953 & 3.89 & -1.12 & -4.56 & -1.29 & -5.08 & 1.16 & 4.51 & -1.28 & -5.01 & -1.1 & -4.37 & -1.18 & -4.83 & -0.582 & -2.68 & -1.13 & -4.65 & 1.06 & 4.22 \\
\hline$\lambda_{\text {cars }}$ & 0.785 & 3.25 & 0.693 & 3.04 & -0.718 & -3.11 & -0.848 & -3.52 & 0.804 & 3.32 & -0.8 & -3.32 & -0.73 & -3.06 & -0.796 & -3.48 & -0.362 & -1.58 & -0.708 & -3.12 & 0.691 & 2.96 \\
\hline$\alpha_{2}$ & -0.0721 & -0.6 & -0.0381 & -0.25 & 0.0237 & 0.11 & -0.127 & -0.64 & -0.0924 & -0.75 & -0.201 & -1.37 & -0.243 & -2.67 & -0.218 & -1.06 & 0.505 & 1.61 & -0.189 & -0.86 & -0.0451 & -0.25 \\
\hline$\alpha_{3}$ & -0.47 & -2.88 & -0.616 & -2.69 & -0.43 & -1.44 & -0.388 & -1.54 & -0.475 & -2.87 & -0.49 & -2.73 & -0.473 & -3.92 & -0.591 & -2.12 & 1.46 & 2.91 & -0.806 & -2.62 & -0.68 & -2.63 \\
\hline$\alpha_{4}$ & -1.29 & -8.33 & -1.41 & -7.12 & -2.1 & -6.6 & -2.03 & -7.22 & -1.32 & -8.23 & -1.58 & -7.72 & -1.14 & -9.33 & -2.19 & -7.45 & -3.33 & -7.43 & -2.41 & -7.55 & -1.42 & -6.3 \\
\hline$\alpha_{5}$ & -1.3 & -10.79 & -1.29 & -7.89 & -2.12 & -6.84 & -1.97 & -7.97 & -1.32 & -10.58 & -1.42 & -9.01 & -1.23 & -13.33 & -2.09 & -8.17 & -0.344 & -0.9 & -2.29 & -8.44 & -1.34 & -6.96 \\
\hline$\alpha_{6}$ & -1.34 & -9.67 & -1.74 & -7.38 & -2.63 & -7.69 & -1.99 & -7.56 & -1.37 & -9.57 & -1.73 & -8.69 & -1.22 & -11.7 & -2.35 & -7.74 & 0.742 & 1.68 & -2.74 & -8.22 & -2.02 & -6.8 \\
\hline$\alpha_{7}$ & -0.534 & -3.93 & -0.488 & -3.04 & -0.75 & -3.02 & -0.758 & -3.35 & -0.55 & -3.96 & -0.463 & -2.67 & -0.391 & -3.63 & -0.789 & -3.31 & -2.3 & -5.51 & -0.781 & -3.1 & -0.542 & -2.94 \\
\hline$\alpha_{8}$ & -0.506 & -3.86 & -0.801 & -4.26 & -1.14 & -3.74 & -0.839 & -3.34 & -0.545 & -3.99 & -0.648 & -3.33 & -0.56 & -5.03 & -1.04 & -3.89 & 1.37 & 5.53 & -1.29 & -4.36 & -0.984 & -4.21 \\
\hline$\alpha_{9}$ & -0.989 & -5.29 & -1.46 & -5.6 & -2.27 & -5.65 & -1.46 & -4.22 & -1 & -5.14 & -1.34 & -5.22 & -0.842 & -5.83 & -2.01 & -5.19 & 0.731 & 2.49 & -2.21 & -5.69 & -1.84 & -5.75 \\
\hline$\gamma_{1}$ & -0.231 & -8.74 & -0.16 & -8.09 & 0.239 & 9.53 & 0.3 & 9.47 & -0.227 & -8.54 & 0.347 & 10.26 & 0.255 & 10.55 & 0.29 & 9.43 & 0.116 & 11.59 & 0.297 & 8.84 & -0.134 & -6.99 \\
\hline$\gamma_{2}$ & -0.134 & -3.3 & -0.112 & -3.58 & 0.139 & 3.81 & 0.187 & 4.17 & -0.141 & -3.47 & 0.181 & 4.04 & 0.158 & 4.59 & 0.193 & 4.36 & 0.0742 & 3.85 & 0.201 & 4.11 & -0.0979 & -3.05 \\
\hline$\gamma_{3}$ & -0.165 & -3.22 & -0.187 & -4.8 & 0.164 & 3.54 & 0.146 & 2.58 & -0.163 & -3.17 & 0.102 & 1.92 & 0.108 & 2.23 & 0.191 & 3.4 & 0.00423 & 0.13 & 0.233 & 3.77 & -0.169 & -4.4 \\
\hline$\gamma_{4}$ & -0.386 & -13.55 & -0.29 & -11.83 & 0.427 & 12.97 & 0.516 & 13.4 & -0.385 & -13.2 & 0.528 & 13.62 & 0.422 & 14.89 & 0.526 & 14 & 0.319 & 30.33 & 0.558 & 13.29 & -0.249 & -10.75 \\
\hline$\gamma_{5}$ & -0.205 & -5.43 & -0.195 & -7.92 & 0.311 & 7.13 & 0.32 & 6.36 & -0.214 & -5.66 & 0.222 & 4.66 & 0.195 & 5.69 & 0.35 & 7.59 & 0.123 & 5.4 & 0.361 & 7.19 & -0.181 & -7.19 \\
\hline$\gamma_{6}$ & -0.24 & -6.17 & -0.305 & -9.5 & 0.421 & 10.59 & 0.352 & 7.03 & -0.239 & -6.02 & 0.357 & 7.11 & 0.218 & 5.67 & 0.421 & 8.31 & 0.051 & 1.77 & 0.495 & 9.48 & -0.319 & -10.79 \\
\hline$\gamma_{n}$ & -0.334 & -11.54 & -0.216 & -9.1 & 0.333 & 10.89 & 0.419 & 11.14 & -0.332 & -11.48 & 0.447 & 11.43 & 0.344 & 12.71 & 0.407 & 10.76 & 0.258 & 16.29 & 0.41 & 9.83 & -0.196 & -9.31 \\
\hline$\gamma_{8}$ & -0.231 & -6.86 & -0.225 & -8.6 & 0.319 & 8.11 & 0.335 & 7.34 & -0.238 & -6.99 & 0.334 & 6.63 & 0.264 & 7.88 & 0.366 & 8.41 & 0.0268 & 1.69 & 0.411 & 8.66 & -0.227 & -8.4 \\
\hline$\gamma_{9}$ & -0.27 & -5.55 & -0.31 & -10.03 & 0.426 & 9.16 & 0.351 & 5.38 & -0.271 & -5.42 & 0.373 & 6.15 & 0.237 & 4.77 & 0.453 & 7.42 & 0.0613 & 3.36 & 0.492 & 8.51 & -0.33 & -11.93 \\
\hline$\sigma_{1}$ & 0.00192 & 0.04 & -0.204 & -4.84 & 0.0325 & 0.72 & 0.206 & 4.25 & 0.00488 & 0.1 & 0.226 & 4.09 & -0.0585 & -1.17 & 0.214 & 4.67 & -1.06 & -25.2 & 0.312 & 7.12 & -0.188 & -4.81 \\
\hline$\sigma_{2}$ & 0.366 & 9.2 & 0.144 & 3.66 & 0.347 & 8.84 & 0.516 & 12.65 & 0.36 & 8.93 & 0.524 & 12.88 & 0.255 & 6.09 & 0.523 & 12.96 & -0.521 & -12.34 & 0.612 & 15.29 & 0.146 & 3.75 \\
\hline$\sigma_{3}$ & 0.38 & 7.45 & 0.127 & 2.31 & 0.366 & 7.43 & 0.565 & 11.9 & 0.384 & 7.56 & 0.551 & 12.04 & 0.365 & 7.77 & 0.556 & 11.3 & -0.365 & -8.29 & 0.631 & 12.49 & 0.0995 & 1.83 \\
\hline$\sigma_{4}$ & -0.262 & -2.58 & -0.338 & -4.04 & 0.00059 & 0.01 & 0.0575 & 0.57 & -0.242 & -2.4 & 0.0482 & 0.47 & -0.344 & -2.81 & 0.0388 & 0.38 & -1.68 & -9.25 & 0.116 & 1.12 & -0.285 & -4.19 \\
\hline$\sigma_{5}$ & 0.025 & 0.4 & -0.313 & -4.71 & 0.159 & 2.29 & 0.288 & 4.21 & 0.0105 & 0.16 & 0.399 & 7.75 & 0.0509 & 0.92 & 0.226 & 3.15 & -0.461 & -7.56 & 0.3 & 4.3 & -0.33 & -5.05 \\
\hline$\sigma_{6}$ & -0.041 & -0.52 & -0.699 & -3.8 & -0.166 & -1.29 & 0.235 & 2.74 & -0.0263 & -0.34 & 0.233 & 2.59 & 0.00644 & 0.09 & 0.0856 & 0.77 & -0.394 & -7.64 & -0.0244 & -0.16 & -0.805 & -3.84 \\
\hline$\sigma_{7}$ & -0.298 & -2.96 & -0.288 & -4.45 & -0.0353 & -0.5 & 0.0831 & 1.01 & -0.272 & -2.79 & 0.105 & 1.2 & -0.25 & -2.92 & 0.134 & 1.79 & -1.23 & -15.52 & 0.251 & 3.63 & -0.313 & -5.32 \\
\hline$\sigma_{8}$ & 0.0532 & 0.87 & -0.148 & -2.35 & 0.28 & 4.63 & 0.397 & 6.54 & 0.0596 & 0.96 & 0.498 & 8.44 & 0.122 & 1.99 & 0.364 & 5.83 & -0.673 & -15.2 & 0.451 & 7.22 & -0.168 & -2.53 \\
\hline$\sigma_{9}$ & 0.109 & 1.27 & -0.416 & -3.29 & 0.105 & 1.04 & 0.427 & 5.15 & 0.101 & 1.16 & 0.4 & 4.57 & 0.18 & 2.34 & 0.283 & 2.61 & -0.675 & -11.56 & 0.29 & 2.68 & -0.532 & -4.01 \\
\hline
\end{tabular}

Table 10: Estimation results (1st part) for the HCMs using the ratings of all evaluators and the median ratings (sample size $=2^{\prime} 265$ ). 


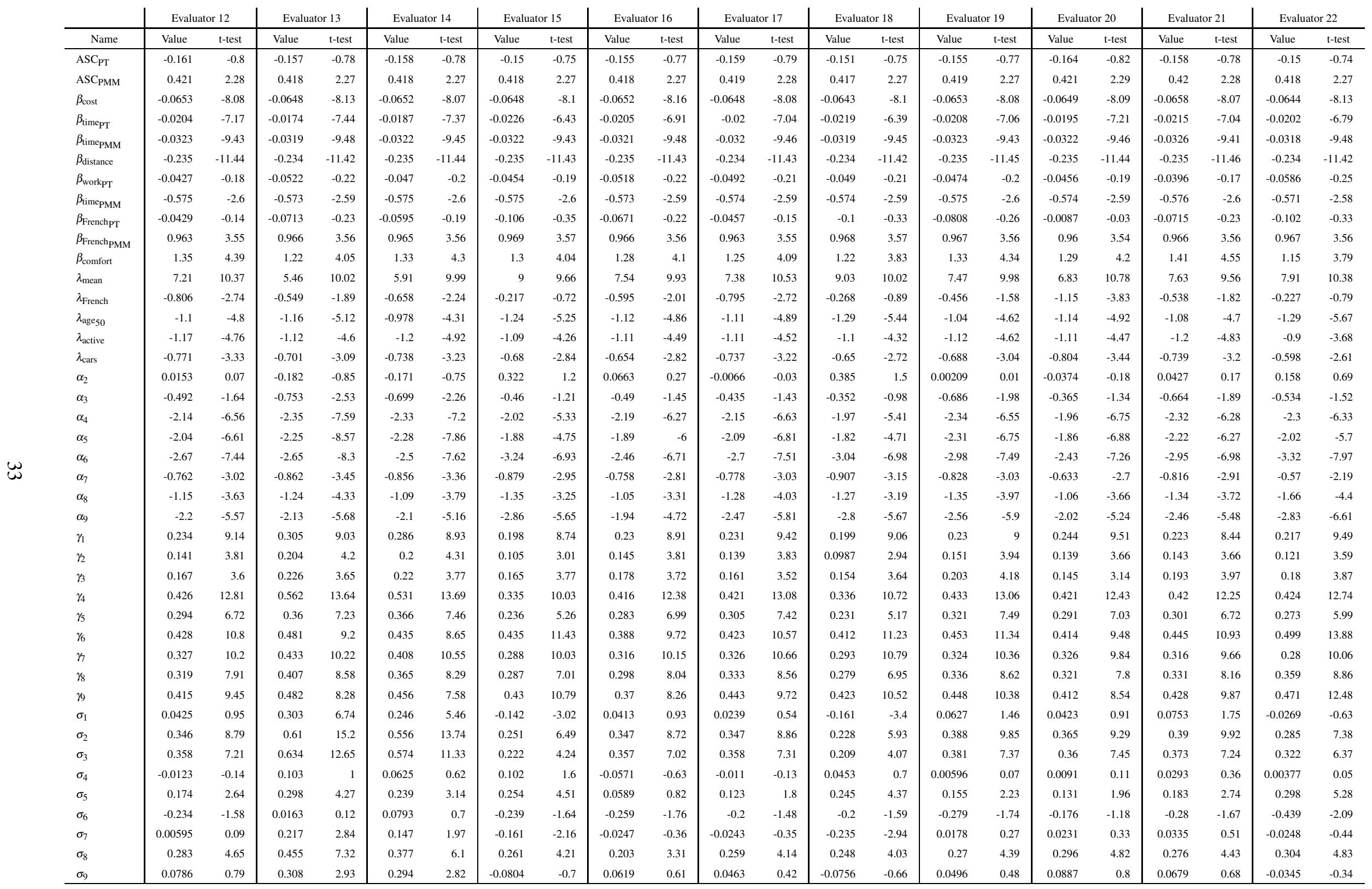

Table 11: Estimation results (2nd part) for the HCMs using the ratings of all evaluators and the median ratings (sample size $=2^{\prime} 265$ ). 


\begin{tabular}{|c|c|c|c|c|c|c|c|c|}
\hline \multirow[b]{2}{*}{ Name } & \multicolumn{2}{|c|}{ Evaluator 23} & \multicolumn{2}{|c|}{ Evaluator 24} & \multicolumn{2}{|c|}{ Evaluator 25} & \multicolumn{2}{|c|}{ Median ratings } \\
\hline & Value & t-test & Value & t-test & Value & t-test & Value & t-test \\
\hline $\mathrm{ASC}_{\mathrm{PT}}$ & -0.152 & -0.76 & -0.126 & -0.63 & -0.123 & -0.61 & -0.155 & -0.77 \\
\hline $\mathrm{ASC}_{\mathrm{PMM}}$ & 0.419 & 2.27 & 0.41 & 2.23 & 0.408 & 2.22 & 0.419 & 2.27 \\
\hline$\beta_{\text {cost }}$ & -0.0653 & -8.1 & -0.0624 & -8.3 & -0.0613 & -8.33 & -0.0653 & -8.08 \\
\hline$\beta_{\text {timePT }}$ & -0.0235 & -6.54 & -0.0179 & -5.18 & -0.0125 & -7.43 & -0.0208 & -7.06 \\
\hline$\beta_{\text {time }_{\text {PMM }}}$ & -0.0323 & -9.42 & -0.0308 & -9.78 & -0.0304 & -9.97 & -0.0323 & -9.43 \\
\hline$\beta_{\text {distance }}$ & -0.235 & -11.44 & -0.232 & -11.37 & -0.232 & -11.36 & -0.235 & -11.45 \\
\hline$\beta_{\text {workpT }_{\text {P }}}$ & -0.0438 & -0.19 & -0.0905 & -0.39 & -0.112 & -0.48 & -0.0474 & -0.2 \\
\hline$\beta_{\text {time }_{\text {PMM }}}$ & -0.576 & -2.6 & -0.562 & -2.54 & -0.556 & -2.51 & -0.575 & -2.6 \\
\hline$\beta_{\text {French }}$ & -0.11 & -0.36 & -0.154 & -0.5 & -0.126 & -0.41 & -0.0808 & -0.26 \\
\hline$\beta_{\text {FrenchpMM }}$ & 0.969 & 3.57 & 0.971 & 3.58 & 0.967 & 3.56 & 0.967 & 3.56 \\
\hline$\beta_{\text {comfort }}$ & 1.36 & 4.26 & 0.687 & 1.97 & 0.0757 & 0.23 & 1.33 & 4.34 \\
\hline$\lambda_{\text {mean }}$ & 9.25 & 9.93 & 8.6 & 9.28 & 0.444 & 1.26 & 7.47 & 9.98 \\
\hline$\lambda_{\text {French }}$ & -0.182 & -0.61 & 0.652 & 2.17 & 0.832 & 2.41 & -0.456 & -1.58 \\
\hline$\lambda_{\text {age }_{50}}$ & -1.08 & -4.67 & -1.21 & -5.08 & 0.746 & 2.83 & -1.04 & -4.62 \\
\hline$\lambda_{\text {active }}$ & -1.14 & -4.55 & -0.348 & -1.32 & 0.476 & 1.69 & -1.12 & -4.62 \\
\hline$\lambda_{\text {cars }}$ & -0.658 & -2.8 & -0.256 & -1.02 & 0.217 & 0.82 & -0.688 & -3.04 \\
\hline$\alpha_{2}$ & 0.31 & 1.18 & 0.354 & 1.19 & 0.295 & 3.48 & 0.00209 & 0.01 \\
\hline$\alpha_{3}$ & -0.48 & -1.24 & -0.343 & -0.71 & 0.721 & 6.3 & -0.686 & -1.98 \\
\hline$\alpha_{4}$ & -2.12 & -5.49 & -2.8 & -5.23 & 0.131 & 1.23 & -2.34 & -6.55 \\
\hline$\alpha_{5}$ & -2.11 & -5.2 & -1.67 & -2.99 & 0.784 & 6.63 & -2.31 & -6.75 \\
\hline$\alpha_{6}$ & -3.29 & -6.97 & -3.42 & -5.78 & 1.05 & 7.53 & -2.98 & -7.49 \\
\hline$\alpha_{7}$ & -0.699 & -2.44 & -0.684 & -1.96 & -0.0106 & -0.13 & -0.828 & -3.03 \\
\hline$\alpha_{8}$ & -1.49 & -3.59 & -1.63 & -3.25 & 0.457 & 4.63 & -1.35 & -3.97 \\
\hline$\alpha_{9}$ & -3.14 & -5.94 & -2.67 & -4.56 & 0.908 & 6.89 & -2.56 & -5.9 \\
\hline$\gamma_{1}$ & 0.195 & 9.02 & 0.218 & 9.21 & 0.193 & 9.08 & 0.23 & 9 \\
\hline$\gamma_{2}$ & 0.106 & 3.24 & 0.146 & 4.16 & 0.0271 & 0.67 & 0.151 & 3.94 \\
\hline$\gamma_{3}$ & 0.169 & 3.94 & 0.203 & 3.83 & -0.102 & -2.28 & 0.203 & 4.18 \\
\hline$\gamma_{4}$ & 0.347 & 11.01 & 0.486 & 10.72 & 0.371 & 13.11 & 0.433 & 13.06 \\
\hline$\gamma_{5}$ & 0.258 & 6.04 & 0.257 & 4.05 & -0.0351 & -0.6 & 0.321 & 7.49 \\
\hline$\gamma_{6}$ & 0.44 & 11.64 & 0.497 & 9.41 & -0.193 & -3.81 & 0.453 & 11.34 \\
\hline$\gamma_{n}$ & 0.262 & 9.85 & 0.289 & 8.53 & 0.311 & 13.68 & 0.324 & 10.36 \\
\hline$\gamma_{8}$ & 0.302 & 7.97 & 0.329 & 6.73 & -0.0028 & -0.06 & 0.336 & 8.62 \\
\hline$\gamma_{9}$ & 0.454 & 11.41 & 0.453 & 8.71 & -0.216 & -4.89 & 0.448 & 10.38 \\
\hline$\sigma_{1}$ & -0.151 & -3.31 & -0.18 & -3.57 & -0.291 & -5.71 & 0.0627 & 1.46 \\
\hline$\sigma_{2}$ & 0.222 & 5.75 & 0.243 & 5.94 & 0.143 & 3.88 & 0.388 & 9.85 \\
\hline$\sigma_{3}$ & 0.209 & 3.99 & 0.356 & 6.67 & 0.208 & 4.34 & 0.381 & 7.37 \\
\hline$\sigma_{4}$ & 0.0361 & 0.53 & 0.146 & 1.46 & -0.427 & -2.78 & 0.00596 & 0.07 \\
\hline$\sigma_{5}$ & 0.228 & 3.93 & 0.61 & 11.89 & 0.272 & 6.25 & 0.155 & 2.23 \\
\hline$\sigma_{6}$ & -0.211 & -1.55 & 0.258 & 2.57 & 0.138 & 2.03 & -0.279 & -1.74 \\
\hline$\sigma_{7}$ & -0.173 & -2.62 & -0.058 & -0.86 & -0.781 & -4.13 & 0.0178 & 0.27 \\
\hline$\sigma_{8}$ & 0.208 & 3.27 & 0.425 & 6.86 & 0.103 & 2.36 & 0.27 & 4.39 \\
\hline$\sigma_{9}$ & -0.144 & -1.12 & 0.183 & 1.75 & 0.0229 & 0.3 & 0.0496 & 0.48 \\
\hline
\end{tabular}

Table 12: Estimation results (3rd part) for the HCMs using the ratings of all evaluators and the median ratings (sample size $\left.=2^{\prime} 265\right)$. 


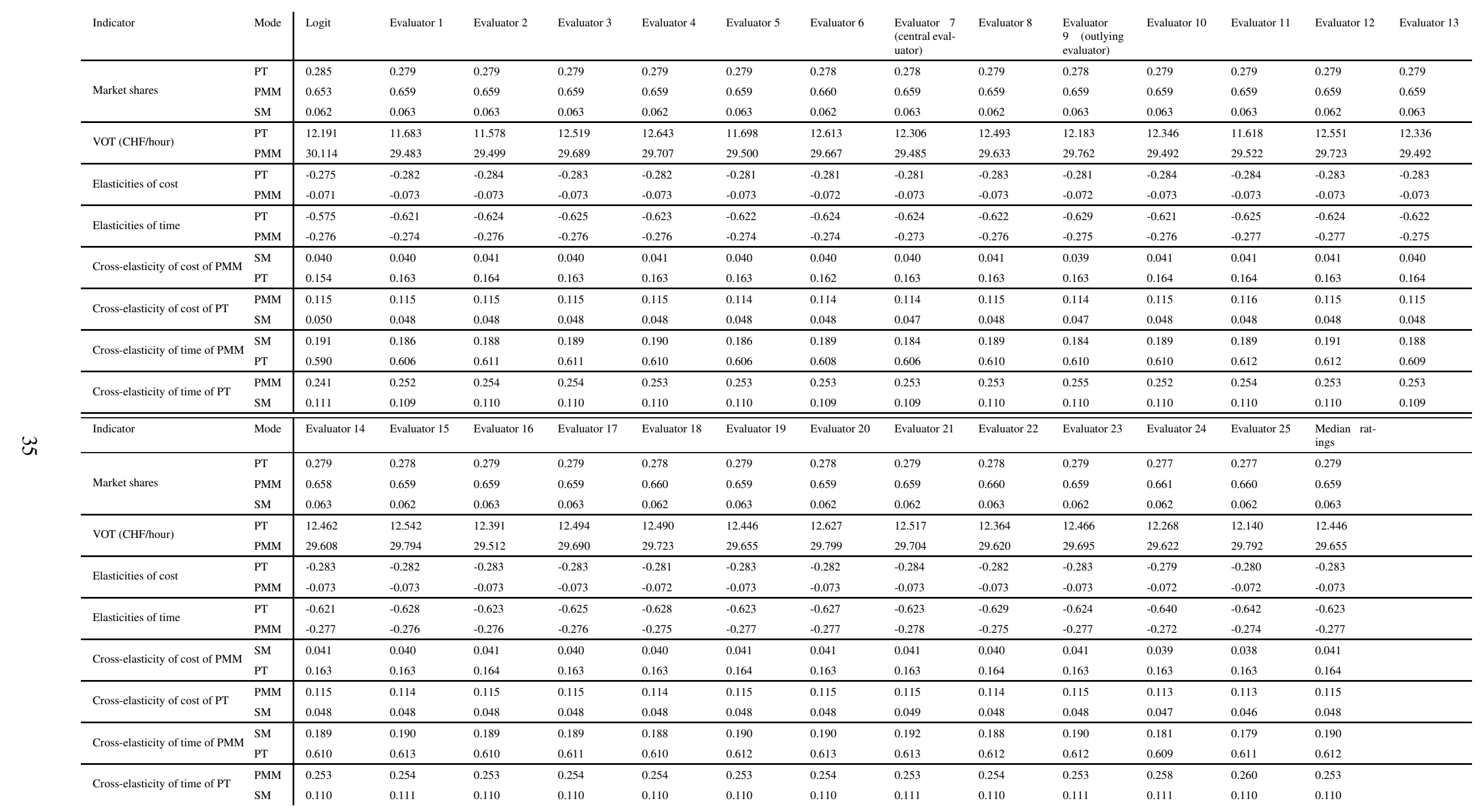

Table 13: Market shares, VOTs, direct and cross-elasticities for the logit model, the models using the ratings of the evaluators and the model using the median ratings (sample size $=2^{\prime} 265$ ). 